\title{
Nutrient Regulation of Endocrine Factors Influencing Feeding and Growth in Fish
}

\author{
Juan Ignacio Bertucci ${ }^{1}$, Ayelén Melisa Blanco ${ }^{1,2}$, Lakshminarasimhan Sundarrajan ${ }^{1}$, \\ Jithine Jayakumar Rajeswari ${ }^{1}$, Cristina Velasco ${ }^{1,2}$ and Suraj Unniappan ${ }^{1 *}$ \\ ${ }^{1}$ Laboratory of Integrative Neuroendocrinology, Department of Veterinary Biomedical Sciences, University of Saskatchewan, \\ Saskatoon, SK, Canada, ${ }^{2}$ Laboratorio de Fisioloxia Animal, Departamento de Bioloxía Funcional e Ciencias da Saúde, \\ Facultade de Bioloxía and Centro de Investigación Mariña, Universidade de Vigo, Vigo, Spain
}

OPEN ACCESS

Edited by:

Encarnación Capilla,

University of Barcelona, Spain

Reviewed by:

Stephane Panserat Institut National de la Recherche

Agronomique (INRA), France

Elisabeth Jönsson Bergman, University of Gothenburg, Sweden

*Correspondence: Suraj Unniappan suraj.unniappan@usask.ca

Specialty section: This article was submitted to Experimental Endocrinology,

a section of the journal

Frontiers in Endocrinology

Received: 16 September 2018

Accepted: 30 January 2019

Published: 28 February 2019

Citation:

Bertucci Jl, Blanco AM, Sundarrajan L, Rajeswari JJ, Velasco C and Unniappan S (2019) Nutrient Regulation of Endocrine Factors Influencing Feeding and Growth in Fish.

Front. Endocrinol. 10:83. doi: 10.3389/fendo.2019.00083
Endocrine factors regulate food intake and growth, two interlinked physiological processes critical for the proper development of organisms. Somatic growth is mainly regulated by growth hormone $(\mathrm{GH})$ and insulin-like growth factors I and II (IGF-I and IGF-II) that act on target tissues, including muscle, and bones. Peptidyl hormones produced from the brain and peripheral tissues regulate feeding to meet metabolic demands. The GH-IGF system and hormones regulating appetite are regulated by both internal (indicating the metabolic status of the organism) and external (environmental) signals. Among the external signals, the most notable are diet availability and diet composition. Macronutrients and micronutrients act on several hormone-producing tissues to regulate the synthesis and secretion of appetite-regulating hormones and hormones of the GH-IGF system, eventually modulating growth and food intake. A comprehensive understanding of how nutrients regulate hormones is essential to design diet formulations that better modulate endogenous factors for the benefit of aquaculture to increase yield. This review will discuss the current knowledge on nutritional regulation of hormones modulating growth and food intake in fish.

Keywords: food intake, growth, nutrients, fish, aquaculture

\section{INTRODUCTION}

Physiological processes in fish, as well as in other vertebrates, are subject to complex regulatory mechanisms that act in response to both internal and external signals (1-4). Signals provided by the environment, along with internal cues are sensed and centrally integrated, providing information about the metabolic status. This enables fish to determine whether conditions are ripe to feed, grow, reproduce and save energy $(4,5)$. Among the external signals that can influence feeding behavior and growth in fish, one of the most important is food $(6,7)$. Both food availability and food composition exert a critical control of these processes, primarily by acting on the hormones in charge of their endocrine control. The main aim of this review is to summarize the recent advances on the role of feeding and fasting, as well as of dietary macro- and micronutrients, on the regulation of appetite- and growth-regulating hormones in fish. A better understanding of the effects of feeding status and diet composition on the expression and release of those hormones could be beneficial to determine the effects of a specific diet or feeding regime on fish health, growth, and development, which could be crucial in aquaculture. This review will also aim to identify gaps in knowledge and directions for future research regarding this important topic. 


\section{NUTRIENTS AND THEIR IMPORTANCE IN FISH}

Nutrients are organic compounds involved in biochemical reactions that produce energy and are constituents of cellular biomass (8). They are divided into two broad groups: macronutrients and micronutrients. Macronutrients are classified into carbohydrates $(\mathrm{CHO})$, proteins and lipids, and are needed in relatively large amounts since they are the primary source to generate the energy organisms require to survive, grow, and reproduce. These nutrients can be stored within the body for later use, or be utilized, leading to the somatic growth of the animal (9). The micronutrients comprise of vitamins and minerals and are needed in smaller quantity, although they have several critical roles in cellular processes. The nutritional requirements regarding the composition and proportion of different nutrients present in the diet vary among species and within species, is determined by various extrinsic and intrinsic factors such as environmental conditions, stage of life cycle, sex, and reproductive state (10). The importance and main roles of each nutrient in fish metabolism are summarized in Table 1.

Organisms coordinate their growth and development with nutrient level fluctuations in their environment, and therefore they must be able to sense their internal and external nutrient levels (24). In mammals, sensing mechanisms regulate specific processes such as food intake, hormone secretion, and energy expenditure to maintain energy homeostasis (25-27). The sensing of nutrients may occur directly or indirectly. In the first case, the nutrient molecule binds to its sensor. In the second case, nutrient abundance is detected through a related molecule (8). These detection processes occur in both central and peripheral tissues in fish. At the central level, the brain integrates metabolic information related to nutrient availability, satiety/hunger signals and hormones related to adiposity. As a result of such signal integration, a response is generated in peripheral tissues aiming to modulate the metabolism $(28,29)$. At the periphery, metabolic regulation by the sensing systems occurs directly or indirectly through the action of endocrine effectors (28).

\section{HORMONES REGULATING FOOD INTAKE}

The regulation of food intake involves the integration of exogenous and endogenous factors to supply the energy necessary to support biological processes. Such regulation is achieved by the endocrine system, which secretes hormones and regulates the activity of the cells by transferring information between the organs. The major organs that secrete hormones involved in regulating appetite are the brain (hypothalamus) and gastrointestinal tissues. The following section will provide a brief description of the main hormones with a critical role in feeding regulation.

\section{Brain Hormones/Neuropeptides Neuropeptide Y (NPY)}

Neuropeptide Y, a $36 \mathrm{kDa}$ amino acid protein, belongs to the NPY family of peptides, which also includes the pancreatic polypeptide (PNP) and peptide tyrosine (PYY). All these peptides share a common three-dimensional structure composed of polyproline coil and an amphipathic helix. The structure of the neuropeptide $\mathrm{Y}$ family is tightened by hydrophobic interactions between prolines and the helix (30). NPY is mainly secreted by the neurosecretory cells in the hypothalamus and is abundantly expressed in the brain, pituitary, spleen, gastrointestinal tract, kidney, testis and smooth muscles $(31,32)$. In teleosts, NPY has been shown to play an essential role in stimulating feeding [see Volkoff (33) for review].

\section{Agouti Related Protein (AgRP)}

AgRP is a 128 amino acid neuropeptide released by the NPY/AgRP neurons, and is an endogenous antagonist of melanocortin receptors 3 and 4 (MC3R and MC4R) (34). It is mainly expressed in the brain, but it is also found in several peripheral tissues, including ovary, muscle and ventral skin (34). AgRP receptor is highly localized at the site of the paraventricular nucleus, the dorsal motor nucleus of the vagus nerve and also in the raphe nucleus, all areas that are highly involved in energy homeostasis. AgRP acts as an orexigenic factor in fish, by antagonizing the activity of MC4R (33).

\section{Proopiomelanocortin (POMC)}

POMC is a 267 amino acid peptide secreted by the hypothalamic neurons located in the arcuate nucleus, as well as the corticotropic cells of the anterior pituitary, the melanotropic cells of the pars intermedia and skin melanocytes (35). In vertebrates, precursors of POMC has three domains, namely $\mathrm{N}$-terminal pro- $\gamma$-melanocyte stimulating hormone (MSH), adrenocorticotropic hormone (ACTH) and C-terminal $\beta$ lipotropin, which are cleaved by the action of prohormone convertases. The most important of these derivatives are $\alpha$ $\mathrm{MSH}$, which plays a vital role in suppressing feeding by acting as an agonist at the anorectic MC4R (33), and ACTH, which regulates the secretion of glucocorticoids from the adrenal glands $(36,37)$.

\section{Cocaine- and Amphetamine-Regulated Transcript (CART)}

CART was isolated from rat striatum upon injection of cocaine and amphetamine, two psychomotor stimulants (38). In goldfish, two forms of CART precursor exist, namely, CARTI that encodes a 117 amino acid pro-CART, and CART-II which encodes a 120 amino acid pro-CART (39). Both CART precursors have been reported to be abundantly expressed in brain, pituitary and also in other peripheral tissues such as eye, interrenal tissues, and gonads in goldfish (39). CART exerts multiple physiological functions in fish, including the inhibition of appetite (39), regulation of the stress response (40) and energy balance (41).

\section{Orexins}

Orexins/hypocretins consist of two orexins, orexin-A, and orexin- $\mathrm{B}$, both cleaved from the same precursor, prepro-orexin $(42,43)$. In fish, both the prepro-orexin RNA and the peptides A and $B$ have been shown to be abundant in the hypothalamus (44), as well as in the gastrointestinal tract (33). Two heptahelical Gprotein coupled receptors are known to mediate orexin functions. Orexins have been reported to have a significant role in increasing feeding behavior and locomotor activity (33), and they were also 
TABLE 1 | The role of principal macronutrients in fish metabolism.

\begin{tabular}{|c|c|c|c|}
\hline Nutrient & Major role in fish & Importance for fish metabolism & References \\
\hline Carbohydrates (CHO) & $\begin{array}{l}\text { Stored as glycogen that can be mobilized to satisfy } \\
\text { energy demands when necessary. In fish, } \mathrm{CHO} \text { seems to } \\
\text { play only a minor role compared to lipids and proteins. } \\
\text { Fish are in general unable to rapidly lower circulating } \\
\text { glucose levels following a glucose load or a high } \mathrm{CHO} \\
\text { meal, thus leading to the interpretation that fish are } \\
\text { glucose-intolerant. }\end{array}$ & $\begin{array}{l}\text { Great importance to the metabolism of all fish species, since they } \\
\text { act as an oxidative substrate to some cells and tissues. } \\
\text { Control of glucose levels exists in tissues relying on glucose such } \\
\text { as the brain. Numerous studies carried out in recent years have } \\
\text { also demonstrated the existence of glucose sensing mechanisms } \\
\text { in such tissues. }\end{array}$ & $(7,11-14)$ \\
\hline Lipids & $\begin{array}{l}\text { Storage and provision of metabolic energy in the form of } \\
\text { ATP provided through the } \beta \text {-oxidation of fatty acids. They } \\
\text { also play an important role as precursors for the } \\
\text { synthesis of many hormones and in the formation of cell } \\
\text { membranes. }\end{array}$ & $\begin{array}{l}\text { Together with their constituent fatty acids, they are the main } \\
\text { nutrients playing important functions as sources of metabolic } \\
\text { energy for growth and reproduction. In fish diet, they are } \\
\text { particularly important the } n-3 \text { and } n-6 \text { unsaturated fatty acids } \\
\text { ("omega 3" and "omega 6"), which are not synthesized by animals, } \\
\text { and therefore must be supplied in the diet. }\end{array}$ & $(15-21)$ \\
\hline Proteins & $\begin{array}{l}\text { Amino acids are an essential component of the diet of all } \\
\text { animals. Fish require a balanced combination of the } 20 \\
\text { amino acids, of which } 10 \text { are not synthesized by fish and } \\
\text { adequate amounts must be provided through their diet. } \\
\text { These essential amino acids are methionine, arginine, } \\
\text { threonine, tryptophan, histidine, isoleucine, lysine, } \\
\text { leucine, valine and phenylalanine. }\end{array}$ & $\begin{array}{l}\text { Proteins and amino acid requirements differ between fish species } \\
\text { since they develop different roles. Some proteins are enzymes, } \\
\text { catalyzing a wide range of chemical reactions; other proteins have } \\
\text { essential functions in muscle contraction, the transport of specific } \\
\text { molecules or as structural elements. In carnivorous fish, proteins } \\
\text { also have an important role as a source of energy. }\end{array}$ & $(9,21,22)$ \\
\hline Vitamins & $\begin{array}{l}\text { Are necessary for normal fish growth and health. They } \\
\text { are usually not synthesized by fish and must be supplied } \\
\text { in the diet. Vitamin deficiency results in scoliosis, dark } \\
\text { coloration and/or, most commonly, reduction in growth } \\
\text { rate. }\end{array}$ & $\begin{array}{l}\text { Are highly important in the proper functioning of the metabolism } \\
\text { since some of them are enzymatic co-factors. }\end{array}$ & (20) \\
\hline Minerals & $\begin{array}{l}\text { Fish can absorb many minerals directly from the water } \\
\text { through their gills and skin, allowing them to compensate } \\
\text { in some degree, mineral deficiencies in their diet. } \\
\text { Some are incorporated into the bones, while others have } \\
\text { a major function in acid-base balance, electron transfer } \\
\text { or the maintenance of cell homeostasis. }\end{array}$ & $\begin{array}{l}\text { They can be divided into two groups based on the quantity } \\
\text { required in the diet. Macro-minerals such as sodium, chloride, } \\
\text { potassium or phosphorous, regulate osmotic balance and are } \\
\text { integrated into the skeletal structure. Micro-minerals are required in } \\
\text { small amounts as components in enzyme and hormone systems. }\end{array}$ & $(20,21,23)$ \\
\hline
\end{tabular}

implicated in the regulation of sleep, energy homeostasis and circadian cycle (45).

\section{Melanin-Concentrating Hormone (MCH)}

Melanin-concentrating hormone (MCH), a 17 amino acid cyclic peptide, was initially isolated from the brain of chum salmon (46). Two genes, $\mathrm{MCH} 1$ and $\mathrm{MCH} 2$, have been identified in zebrafish and pufferfish (47). The MCH receptor was identified as the GPCR SLC-1, later termed as MCH-R1. MCH-R1 couples to different G-proteins and plays an essential role in activating different signaling pathways. The MCH-R1 is preferentially expressed in the brain, particularly in the hypothalamus, areas of the cortex, arcuate and ventromedial nuclei, and olfactory lobes. $\mathrm{MCH}$ mainly acts on the melanophores regulating the color change and also lightens the skin in fish. Besides this, $\mathrm{MCH}$ seems to have a role in the regulation of feeding, although such a function is still unclear in fish [see Volkoff (33) for review].

\section{Nesfatin-1}

Nesfatin-1 is an 82 amino acid peptide originally isolated from the rat hypothalamus and is encoded in the nucleobindin-2 (NUCB2) gene (48). It is proposed that NUCB2 is cleaved by the prohormone convertases into three different peptides, namely nesfatin-1 (82 amino acids), nesfatin-2 (85-163 amino acids) and nesfatin-3 (166-396 amino acids), respectively. Of all these peptides, nesfatin-1 has been shown to have biological activity. The 30-amino acid mid-segment of nesfatin1 is considered as the bioactive core and has been shown to affect appetite, the hypothalamus-pituitary-ovarian axis, and to modulate intracellular $\mathrm{Ca}_{2}{ }^{+}$signaling in mammals (49). Among non-mammals, nesfatin-1 has been studied in various fish species, including goldfish (50), Ya-fish (51), and rainbow trout (52). In goldfish, nesfatin-1-like immunoreactivity has been found in the hypothalamus, particularly in the nucleus lateralis tuberis (NLT) (50). Exogenous administration of nesfatin-1 has been shown to cause anorectic actions in goldfish (50).

\section{Hormones Primarily Arising From Peripheral Tissues Ghrelin}

Ghrelin has been identified in numerous fish species. It consists of 28 amino acids in mammals and has 12 to 25 amino acids in fish depending on the species. The gene encoding the protein was identified within the chromosome 3 and consists of four exons. Ghrelin exerts its physiological functions by binding to the growth hormone secretagogue receptor-1a (GHS-R1a/ghrelin receptor) (53). This peptide is mainly released in the stomach 
(or its equivalent in stomachless species), although other tissues have been shown to synthesize the hormone, particularly the hypothalamus (54). The central role described for ghrelin is its potent orexigenic role (33), but there are other well-known physiological roles for this hormone, including the regulation of $\mathrm{GH}$ release from the pituitary, a role in energy balance regulation, and cardiovascular effects, among others (55-57).

\section{Cholecystokinin (CCK)}

CCK is a peptide characterized by a C-terminal tetrapeptide sequence. The structure of pro-CCK consists of three sulfated tyrosine residues, which play a crucial role in the activation of CCK receptors (58). CCK binds to two receptor subtypes, CCK$A$ receptor (CCK1) and CCK-B receptor (CCK2), which are primarily localized at the site of the gastrointestinal tract and the brain (59). In fish, CCK and its cleavage sites suggest that the precursor protein (pro-CCK) is processed into octapeptides and are fully sulfated. CCK plays an essential role in the regulation of feeding, influences digestion and activates satiety signals (60). In goldfish, acute administration of CCK resulted in the suppression of food intake (61), likely by acting on NPY and orexin-A (62).

\section{Peptide YY}

Peptide YY, a member of the Y family of peptides, is a 36 amino acid gut-brain hormone that is known to have anorectic actions in goldfish (63). PYY is released from the endocrine cells of ileum and colon (64), and binds to the NPY receptor 1 which is abundantly expressed in the brain and gut of fish. Similar to NPY, PYY plays an essential role in signaling between the enteric nervous system and central nervous system in fish $(31,65)$.

\section{Glucagon-Like-Peptide-1 (GLP-1)}

GLP-1, another anorexigenic intestinal peptide, belongs to the family of glucagon-like peptides encoded in the preproglucagon gene. In fish, the pancreas synthesizes glucagon and GLP-1, while the intestine secretes oxyntomodulin (66), and all of these peptides are processed from the proglucagon in the nervous system and intestine. GLP mRNAs have been identified in several teleosts, and its receptor (GLP-1R) has been successfully cloned in zebrafish and goldfish (67). Apart from reducing food intake, GLP-1 has been involved in gastric emptying and plays an important role in regulating liver glycogenolysis and gluconeogenesis (66).

\section{Leptin}

Leptin is a $16 \mathrm{kDa}$ protein encoded in chromosome 7 . While mammalian leptin is mainly produced by the adipose tissue, the liver appears to be the main leptin production site in fish (68). The structure of leptin resembles that of growth hormone, belonging to the family of tetrahelical cytokines. In fish, this hormone has been shown to affect adipogenesis (57), and to increase lipolysis while reducing lipogenesis in liver (69). Besides this, leptin decreases food intake in several fish species (33), likely by stimulating the anorexigenic neuropeptides POMC and CART (70).

\section{HORMONAL REGULATORS OF GROWTH}

Regulation of growth in fish, as well as in most of the vertebrates, is coordinated by the GH-IGF system (71). A summary of each component of the GH-IGF system as well as their principal function related to growth is presented in this section.

\section{Growth Hormone}

Growth hormone $(\mathrm{GH})$ is an endocrine regulator of many physiological processes in vertebrates. In fish, GH is involved in almost all physiological processes including osmotic balance, lipid, protein and $\mathrm{CHO}$ metabolism, reproduction and growth. Moreover, studies have indicated that $\mathrm{GH}$ also affects behavioral aspects, such as appetite (72) and foraging (73) in rainbow trout and transgenic Atlantic salmon, respectively. In fish, $\mathrm{GH}$ is released from the adenohypophysis in response to hypothalamic signals, and exerts its effects on target tissues (74).

\section{Growth Hormone Receptors}

In fish cells, GH binds to its receptors GHR-I and GHRII (growth hormone receptor I and II, respectively) to exert its biological actions (75). GH receptors (GHRs) are widely distributed in tissues, but the primary expression is in the liver (or hepatopancreas). In that tissue, the important response to $\mathrm{GH}$ binding its receptors is the release of the insulin-like growth factor I (IGF-I). In other tissues, GHRs also mediate the growth-promoting effects of $\mathrm{GH}$, although the liver is still the place in which GHRs have a significant role in the somatic growth regulation.

\section{Insulin-Like Growth Factors}

As GHRs, IGF-I and IGF-II are expressed in several tissues, but the main expression is in fish liver. Both factors play a key role in the promotion of cellular proliferation and differentiation in vertebrates (76-78). These and other biological functions of IGFs are mediated by binding to specific transmembrane receptors, present in fish as well as in mammals (79). Apart from growth, IGF-I has also been associated with fish metabolism, development, reproduction and osmoregulation in seawater (74). The IGF-II mRNA has been detected in the liver as well as in the brain, heart, kidney, gills, gastrointestinal tract, pancreatic islets, skeletal muscle and gonads of fish (74). The widespread gene expression of IGF-II detected by RT-qPCR in both juvenile and adult fish contrasts the findings in mammals, in which its expression seems to be relevant only during the early stages of development (80). A role in metabolism regulation by IGF-II was demonstrated in muscle cells from trout (81), indicating that this factor could act as a metabolic hormone in fish. There is evidence that $\mathrm{GH}$ regulates igf-II gene in all tissues in fish (82-84), while GH most likely regulates only the expression of the igf-I gene in vertebrates. This situation makes fish an excellent model to study species-specific differences in the growth system. 


\section{NUTRITIONAL REGULATION OF HORMONES MODULATING FOOD INTAKE IN FISH}

\section{Feeding}

The nutritional status is an important modulator of appetiteregulating hormones in fish. In this respect, several central and peripheral appetite regulators are affected by a single meal, showing periprandial fluctuations in their expression and/or secretion levels. Brain hormones showing such changes in fish include NPY $(85,86)$, orexin (87-90), CART $(87,88,91,92)$, and nesfatin-1 (93). In general terms, appetite-stimulating or orexigenic factors, including NPY $(85,86)$, and orexin $(89,90)$, have been shown to display periprandial changes characterized by higher expression levels before or at mealtime, and lower levels post feeding, suggesting that they are hunger signals. By contrast, appetite inhibiting or anorexigenic signals, such as CART (91, 92), were found to be mainly upregulated after a meal, thus acting as a postprandial satiety signal.

As for peripheral appetite-regulating hormones, the most studied in terms of periprandial fluctuations have been ghrelin, CCK, PYY, and leptin. Circulating levels of ghrelin (in its acylated form) were found to rise pre-prandially in goldfish (94), supporting the role of acyl-ghrelin as a meal initiator in this teleost. Consistently, Unniappan et al. (95) described a significant decrease in total ghrelin in circulation after a meal in goldfish, although this postprandial decrease in plasma ghrelin levels seems to be species-specific as it was detected neither in rainbow trout (96) nor in Mozambique tilapia (97). Ghrelin has also been shown to display periprandial fluctuations in terms of gene expression, although different profiles have been described depending on the species [Atlantic cod, (90); gibel carp, (98); goldfish, (94, 95); Mozambique tilapia, (97, 99); Schizothorax prenanti, (100); zebrafish, (101)]. The periprandial changes of CCK have been only studied in terms of its mRNA expression. Although species-specific changes were observed $(88,89)$, periprandial variations of $c c k$ expression are mainly characterized by an increase in mRNA levels after a meal in the intestine and/or the hypothalamus [dourado, (87); Schizottorax prenanti, (102)]. A similar periprandial profile was described for pyy in the hypothalamus of the Mexican blind cavefish (89) and Schizottorax prenanti (103), in the brain of goldfish (63), and in the brain and gut of Siberian sturgeon (104). Finally, leptin seems only to vary postprandially, although such variations are species- and tissue-specific. Thus, postprandial increases in leptin mRNA expression has been observed in the brain and liver of the mandarin fish (105), in the liver of goldfish (106) and in the visceral adipose tissue of the Atlantic salmon (107), but not in the brain of goldfish (106), the brain and intestine of pacu (88), or the liver of Schizothorax prenanti (102).

\section{Food Deprivation}

Food deprivation has been shown to regulate the secretion and expression of appetite-regulating hormones. Table 2 includes a summary of the available studies within the literature and shows the main effect of fasting periods of different duration on the circulating levels and the mRNA expression of the main appetite regulators in several fish species. In general terms, as expected, fasting has been found to upregulate the levels of orexigens and decrease the levels of anorexigens, but several exceptions have been observed depending on the duration of the fasting period, the tissue analyzed and the species (see Table 2 for results and references). In general an upregulation of orexigens and the GH-IGF system should result in an increase in growth. However, without a complete profile of the redundant endocrine milieu, such conclusions are not valid. In addition, a major limitation of many of the studies is that only mRNA expression was determined. Without understanding more about the peptide synthesis in its major tissue sources, and its levels in circulation, it is difficult to reach conclusions on the effects of nutrient status on these hormones.

\section{Diet Composition}

Another nutritional aspect influencing the appetite-regulating hormones is the composition of diets. This is of great importance as there is significant interest in fisheries and aquaculture in modulating fish growth and reproduction by altering diet and/or endocrine milieu. Therefore, it is important to understand the dietary regulation of hormones, as they have remarkable effects on both reproduction and growth. Although the literature available on this is not very large, several studies have described that altering the macronutrient (i.e., carbohydrates, proteins, and fat) composition of the diet has significant effects on the secretion and/or expression of appetite-regulating hormones in fish.

\section{Carbohydrates (CHO)}

Few studies have been performed in fish describing the effects of $\mathrm{CHO}$ on appetite regulators. In 2002, Narnaware and Peter described that feeding goldfish $\mathrm{CHO}$-enriched diets significantly reduces NPY mRNA levels in brain areas (175). Additionally, this macronutrient has been shown to upregulate preproghrelin mRNA expression in the pituitary (176). An in vitro assay performed to test the effects of glucose on the expression of appetite-regulating hormones revealed that exposure of goldfish intestinal fragments to different concentrations of this monosaccharide leads to a downregulation of nucb2/nesfatin-1 mRNAs and an upregulation of preproghrelin mRNA expression (177).

\section{Proteins}

High-protein diets also modulate important appetite-regulating hormonal systems. A study performed in sea bream revealed that fish fed on high protein diets has higher mRNA expression levels of preproghrelin and cck than those fish fed on diets containing a lower amount of protein (178). In goldfish, feeding diets enriched in this type of macronutrient results in a significant increase in nucb2/nesfatin-1 expression in the pituitary, and a significant decrease in nucb2/nesfatin-1 mRNAs in the gut and in preproghrelin mRNAs in the liver (176). Accordingly, expression of both nucb2/nesfatin-1 and preproghrelin was reduced by direct exposure of goldfish intestinal and hepatopancreas fragments to L-tryptophan (177). Very recently, Volkoff et al. demonstrated that the replacement of dietary fish protein with soy protein does not produce major changes in the expression of cart, orexin, $c c k$, 
TABLE 2 | Effects of fasting periods of different duration on the circulating levels and the mRNA expression of the main appetite-regulating hormones in several fish species.

\begin{tabular}{|c|c|c|c|c|c|c|c|c|c|}
\hline & \multicolumn{3}{|c|}{$\begin{array}{l}\text { Short-term fasting } \\
\text { (1-6 days) }\end{array}$} & \multicolumn{3}{|c|}{$\begin{array}{l}\text { Mid-term fasting } \\
\text { (7-29 days) }\end{array}$} & \multicolumn{3}{|c|}{$\begin{array}{l}\text { Long-term fasting } \\
\text { ( } \geq 30 \text { days) }\end{array}$} \\
\hline & $\begin{array}{l}\text { Circulating } \\
\text { levels }\end{array}$ & Brain expres. & $\begin{array}{l}\text { Periph. } \\
\text { expres. }\end{array}$ & $\begin{array}{l}\text { Circulating } \\
\text { levels }\end{array}$ & Brain expres. & $\begin{array}{l}\text { Periph. } \\
\text { expres. }\end{array}$ & $\begin{array}{l}\text { Circulating } \\
\text { levels }\end{array}$ & $\begin{array}{l}\text { Brain } \\
\text { expres. }\end{array}$ & $\begin{array}{l}\text { Periph. } \\
\text { expres. }\end{array}$ \\
\hline NPY & & $\begin{array}{l}\text { Atlantic salmon }=(108) \\
\text { Blunt snout bream } \uparrow \\
\text { (109) } \\
\text { Goldfish } \uparrow(110)\end{array}$ & $\begin{array}{l}\text { Blunt snout } \\
\text { bream = (109) }\end{array}$ & & $\begin{array}{l}\text { Blunt snout bream } \uparrow \\
(109) \\
\text { Brazilian flounder } \uparrow(85) \\
\text { Coho salmon } \uparrow(111) \\
\text { Cunner } \downarrow \text { (112) } \\
\text { Nile tilapia } \uparrow(113) \\
\text { Platyfish = (114) } \\
\text { Schizothorax prenanti } \\
\uparrow(115) \\
\text { Tiger puffer } \uparrow(116) \\
\text { Winter flounder } \uparrow(117) \\
\text { Winter skate } \uparrow(118) \\
\text { Yellowtail } \uparrow(119) \\
\text { Zebrafish } \uparrow(120)\end{array}$ & $\begin{array}{l}\text { Blunt snout } \\
\text { bream = (109) }\end{array}$ & & $\begin{array}{l}\text { Rainbow } \\
\text { trout = (121) }\end{array}$ & \\
\hline POMC & & Flatfish $\uparrow(126)$ & & & $\begin{array}{l}\text { Goldfish }=(127) \\
\text { Rainbow trout }=\uparrow \\
\text { (128) } \\
\text { Zebrafish }=(125) \\
\text { Zebrafish } \downarrow(129)\end{array}$ & & & $\begin{array}{l}\text { Rainbow trout } \\
\uparrow(121)\end{array}$ & \\
\hline CART & & $\begin{array}{l}\text { Atlantic salmon } \downarrow \text { (108) } \\
\text { Catfish } \downarrow \text { (130) } \\
\text { Dourado }=(87) \\
\text { Goldfish } \downarrow \text { (39) } \\
\text { Siberian sturgeon } \uparrow \\
\text { (92) } \\
\text { Zebrafish } \downarrow \text { (131) }\end{array}$ & & & $\begin{array}{l}\text { Atlantic cod } \downarrow \text { (132) } \\
\text { Channel catfish } \downarrow \text { (133) } \\
\text { Common carp } \downarrow \text { (124) } \\
\text { Cunner } \downarrow \text { (112) } \\
\text { Goldfish } \downarrow \text { (134) } \\
\text { Pacu } \downarrow \text { (88) } \\
\text { Platyfish } \downarrow \text { (114) } \\
\text { Red-bellied piranha } \downarrow \\
\text { (135) } \\
\text { Schizothorax prenanti } \\
\downarrow \text { (91) } \\
\text { Siberian sturgeon } \uparrow(92) \\
\text { Winter flounder } \downarrow \text { (117) } \\
\text { Winter skate }=(118)\end{array}$ & & & $\begin{array}{l}\text { Rainbow } \\
\text { trout }=(121)\end{array}$ & \\
\hline Orexin & & $\begin{array}{l}\text { Dourado } \uparrow(87) \\
\text { Zebrafish = }(136)\end{array}$ & & & $\begin{array}{l}\text { Blind cave fish } \uparrow \text { (89) } \\
\text { Cunner } \downarrow \text { (112) } \\
\text { Goldfish } \uparrow(134) \\
\text { Pacu } \uparrow(88) \\
\text { Platyfish } \uparrow(114) \\
\text { Red-bellied piranha } \uparrow \\
\text { (135) } \\
\text { Zebrafish } \uparrow(136,137)\end{array}$ & & & & \\
\hline $\mathrm{MCH}$ & & Starry flounder $\uparrow(138)$ & & & $\begin{array}{l}\text { Atlantic code } \uparrow(139) \\
\text { Barfin flounder } \uparrow(140) \\
\text { Hammerhead } \\
\text { shark }=(141) \\
\text { Schizothorax prenanti } \\
\uparrow(142) \\
\text { Winter flounder } \uparrow(143) \\
\text { Zebrafish } \uparrow(144)\end{array}$ & & & & \\
\hline
\end{tabular}


TABLE 2 | Continued

\begin{tabular}{|c|c|c|c|c|c|c|c|c|c|}
\hline & \multicolumn{3}{|c|}{$\begin{array}{l}\text { Short-term fasting } \\
\text { (1-6 days) }\end{array}$} & \multicolumn{3}{|c|}{$\begin{array}{l}\text { Mid-term fasting } \\
\text { (7-29 days) }\end{array}$} & \multicolumn{3}{|c|}{$\begin{array}{l}\text { Long-term fasting } \\
\text { ( } \geq 30 \text { days) }\end{array}$} \\
\hline & $\begin{array}{l}\text { Circulating } \\
\text { levels }\end{array}$ & Brain expres. & $\begin{array}{l}\text { Periph. } \\
\text { expres. }\end{array}$ & $\begin{array}{l}\text { Circulating } \\
\text { levels }\end{array}$ & Brain expres. & $\begin{array}{l}\text { Periph. } \\
\text { expres. }\end{array}$ & $\begin{array}{l}\text { Circulating } \\
\text { levels }\end{array}$ & $\begin{array}{l}\text { Brain } \\
\text { expres. }\end{array}$ & $\begin{array}{l}\text { Periph. } \\
\text { expres. }\end{array}$ \\
\hline Nesf-1 & & Zebrafish = (93) & $\begin{array}{l}\text { Goldfish } \uparrow \\
(50) \\
\text { Ya-fish } \uparrow(51) \\
\text { Zebrafish = (93) }\end{array}$ & & $\begin{array}{l}\text { Goldfish } \downarrow \text { (50) } \\
\text { Ya-fish } \downarrow \text { (51) } \\
\text { Zebrafish } \downarrow \text { (93) }\end{array}$ & $\begin{array}{l}\text { Goldfish } \uparrow \\
\text { (50) } \\
\text { Ya-fish } \uparrow(51) \\
\text { Zebrafish } \downarrow \\
\text { (93) }\end{array}$ & & & \\
\hline Ghrelin & $\begin{array}{l}\text { Atlantic } \\
\text { salmon } \uparrow \\
(145) \\
\text { Tilapia }=(97)\end{array}$ & $\begin{array}{l}\text { Blunt snout bream } \uparrow \\
\text { (109) } \\
\text { Grass carp } \uparrow(146) \\
\text { Schizothorax } \\
\text { prenanti }=(100) \\
\text { Zebrafish }=(147) \\
\text { Zebrafish } \uparrow(148)\end{array}$ & $\begin{array}{l}\text { Atlantic } \\
\text { salmon } \uparrow \\
(149) \\
\text { Atlantic } \\
\text { salmon } \downarrow \\
\text { (145) } \\
\text { Blunt snout } \\
\text { bream } \uparrow(109) \\
\text { Chinese } \\
\text { perch } \downarrow \text { (150) } \\
\text { Gibel } \\
\text { carp }=(98) \\
\text { Grass carp } \uparrow \\
\text { (146) } \\
\text { Schizothorax } \\
\text { davidi } \uparrow(151) \\
\text { Schizothorax } \\
\text { prenanti }=(100) \\
\text { Sea } \\
\text { bass }=(152) \\
\text { Tilapia }=(97) \\
\text { Zebrafish } \downarrow \\
\text { (153) } \\
\text { Zebrafish } \uparrow \\
\text { (148) }\end{array}$ & $\begin{array}{l}\text { Atlantic } \\
\text { salmon }=(145) \\
\text { Goldfish }= \\
\text { (93) } \\
\text { Hybrid striped } \\
\text { bass } \uparrow(154) \\
\text { Rainbow } \\
\text { trout }=(96) \\
\text { Tilapia }=(155) \\
\text { Tilapia } \uparrow(97) \\
\end{array}$ & $\begin{array}{l}\text { Blunt snout bream } \uparrow \\
\text { (109) } \\
\text { Goldfish } \\
=\uparrow(93,156) \\
\text { Grass carp } \uparrow(146) \\
\text { Red-bellied } \\
\text { piranha }=(157) \\
\text { Zebrafish }=(147) \\
\text { Zebrafish } \uparrow(148)\end{array}$ & $\begin{array}{l}\text { Atlantic } \\
\text { salmon }=(145) \\
\text { Blunt snout } \\
\text { bream } \uparrow(109) \\
\text { Gibel carp } \uparrow \\
\text { (98) } \\
\text { Goldfish } \uparrow \\
(93,156) \\
\text { Grass carp } \uparrow \\
\text { (146) } \\
\text { Red-bellied } \\
\text { piranha } \uparrow \\
\text { (157) } \\
\text { Schizothorax } \\
\text { davidi } \uparrow(151) \\
\text { Sea bass } \uparrow \\
\text { (152) } \\
\text { Tilapia }=(97) \\
\text { Zebrafish } \uparrow \\
\text { (148) }\end{array}$ & & & \\
\hline PYY & & $\begin{array}{l}\text { Goldfish } \downarrow \text { (63) } \\
\text { Siberian sturgeon } \downarrow \\
\text { (104) } \\
\text { Schizothorax prenanti } \\
\downarrow \text { (103) }\end{array}$ & $\begin{array}{l}\text { Siberian } \\
\text { sturgeon } \downarrow \\
(104) \\
\text { Atlantic } \\
\text { salmon }=(149) \\
\text { Yellowtail } \uparrow \\
(160)\end{array}$ & & $\begin{array}{l}\text { Blind cave fish }=(89) \\
\text { Goldfish } \downarrow \text { (63) } \\
\text { Nile tilapia } \downarrow \text { (113) } \\
\text { Red-bellied } \\
\text { piranha }=(135) \\
\text { Siberian sturgeon } \downarrow \\
\text { (104) } \\
\text { Schizothorax prenanti } \\
\downarrow(103)\end{array}$ & $\begin{array}{l}\text { Nile tilapia } \downarrow \\
\text { (113) } \\
\text { Red-bellied } \\
\text { piranha } \downarrow \\
\text { (135) } \\
\text { Siberian } \\
\text { sturgeon } \downarrow \\
\text { (104) }\end{array}$ & & & \\
\hline
\end{tabular}


TABLE 2 | Continued

\begin{tabular}{|c|c|c|c|c|c|c|c|c|c|}
\hline & \multicolumn{3}{|c|}{$\begin{array}{l}\text { Short-term fasting } \\
\text { (1-6 days) }\end{array}$} & \multicolumn{3}{|c|}{$\begin{array}{l}\text { Mid-term fasting } \\
\text { (7-29 days) }\end{array}$} & \multicolumn{3}{|c|}{$\begin{array}{l}\text { Long-term fasting } \\
\text { ( } \geq 30 \text { days) }\end{array}$} \\
\hline & $\begin{array}{l}\text { Circulating } \\
\text { levels }\end{array}$ & Brain expres. & $\begin{array}{l}\text { Periph. } \\
\text { expres. }\end{array}$ & $\begin{array}{l}\text { Circulating } \\
\text { levels }\end{array}$ & Brain expres. & $\begin{array}{l}\text { Periph. } \\
\text { expres. }\end{array}$ & $\begin{array}{l}\text { Circulating } \\
\text { levels }\end{array}$ & $\begin{array}{l}\text { Brain } \\
\text { expres. }\end{array}$ & $\begin{array}{l}\text { Periph. } \\
\text { expres. }\end{array}$ \\
\hline Leptin & & Mandarin fish $\uparrow(105)$ & $\begin{array}{l}\text { Common } \\
\text { carp }=(161) \\
\text { Mandarin fish } \\
\uparrow(105) \\
\text { Schizothorax } \\
\text { prenanti } \downarrow \\
(102)\end{array}$ & $\begin{array}{l}\text { Fine flounder } \\
\uparrow(162,163) \\
\text { Rainbow trout } \\
\uparrow(164) \\
\text { Tilapia } \uparrow(1)\end{array}$ & $\begin{array}{l}\text { Goldfish }=(106) \\
\text { Orange-spotted } \\
\text { grouper }=(165) \\
\text { Pacu }=(88) \\
\text { Red-bellied } \\
\text { piranha }=(157) \\
\text { Tilapia }=(166)\end{array}$ & $\begin{array}{l}\text { Common } \\
\text { carp }=(161) \\
\text { European sea } \\
\text { bass } \uparrow(167) \\
\text { Goldfish }=(106) \\
\text { Green sunfish } \\
\downarrow \text { (168) } \\
\text { Orange- } \\
\text { spotted } \\
\text { grouper } \uparrow \\
\text { (165) } \\
\text { Pacu }=(88) \\
\text { Rainbow trout } \\
\uparrow(169) \\
\text { Red-bellied } \\
\text { piranha } \downarrow \\
\text { (157) } \\
\text { Schizothorax } \\
\text { prenanti } \downarrow \\
\text { (102) } \\
\text { Striped bass } \\
\downarrow \text { (170) } \\
\text { Tilapia } \uparrow(1) \\
\text { White-clouds } \\
\text { Mountain } \\
\text { minnow } \uparrow \\
(171) \\
\text { Zebrafish } \downarrow \\
\text { (172) }\end{array}$ & $\begin{array}{l}\text { Fine } \\
\text { flounder }=(163) \\
\text { Rainbow } \\
\text { trout }=(121) \\
\text { ) }\end{array}$ & & $\begin{array}{l}\text { Arctic charr } \uparrow \\
(173) \\
\text { Atlantic } \\
\text { salmon }=(107) \\
\text { Eel }=(174) \\
\text { Rainbow trout } \\
\uparrow(121)\end{array}$ \\
\hline
\end{tabular}

$=$ levels not altered; $\uparrow$ levels upregulated; $\downarrow$ levels downregulated.

and leptin in the pacu (88). Similarly, varying the diet lysine to arginine ratio has been described to not significantly modify the expression of $n p y$ and $c c k$ in juvenile cobia (86). Leucine reduced leptin secretion from the adipocytes of food-restricted rainbow trout (57).

\section{Lipids}

Intake of fat-enriched diets has been described to reduce the gene expression of $n p y$ in the goldfish telencephalon-preoptic area (175), and to increase the mRNA levels of nucb2/nesfatin-1 in the hypothalamus and liver, and of preproghrelin in the pituitary of goldfish (176). Treatment of goldfish intestine with different fatty acids in vitro revealed that fatty acids, in general, downregulate NUCB2/nesfatin-1 in the intestine, but only the longer and highly unsaturated fatty acids inhibit preproghrelin (177). Jönsson and coworkers showed that rainbow trout fed a normal-protein/highlipid diet tends to have higher plasma ghrelin levels than those fed a high-protein/low-lipid diet (96). Apart from the abovementioned studies, which were all carried out in adult fish, few studies have been performed at larvae or post-larvae state to study the effects of the replacement of the dietary fat source on the expression of metabolic hormones. Bertucci and coworkers demonstrated that the replacement of dietary fish oil with sunflower oil leads to a decrease in nucb2/nesfatin-1 mRNA expression in pejerrey larvae (179). Additionally, it was described that Senegalese sole post-larvae fed with diets containing soybean oil have higher cart1 and cck mRNA levels in the brain, but lower peripheral $c c k$ levels than larvae fed cod liver oil (180).

\section{NUTRITIONAL REGULATION OF THE GH-IGF SYSTEM AND ITS INFLUENCE ON FISH GROWTH}

\section{Nutritional Status}

The main environmental factor that regulates the GH-IGF system is the nutritional status (181). Table 3 summarizes the effects of fasting on the expression of components of the GH-IGF system in different fish species. During fasting, growth ceases, and energy is mobilized from tissues to support metabolism. This is mainly mediated by changes in the GH-IGF system: plasma levels of GH generally increase while plasma levels of IGFs decrease (182). These changes are explained by a phenomenon known as liver GH resistance, which is characterized by the fact that hepatocytes become resistant to $\mathrm{GH}$, resulting in decreased IGF production despite elevated GH (197). These changes in GHIGF system during fasting could be adaptive jn response to the 
TABLE 3 | Effects of fasting periods of different duration on the circulating levels and the mRNA expression of the GH-IGF system endocrine components in several fish species.

\begin{tabular}{|c|c|c|c|c|c|c|c|c|c|}
\hline & \multicolumn{3}{|c|}{$\begin{array}{l}\text { Short-term fasting } \\
\text { (1-6 days) }\end{array}$} & \multicolumn{3}{|c|}{$\begin{array}{l}\text { Mid-term fasting } \\
\text { (7-29 days) }\end{array}$} & \multicolumn{3}{|c|}{$\begin{array}{l}\text { Long-term fasting } \\
\text { ( } \geq 30 \text { days) }\end{array}$} \\
\hline & $\begin{array}{l}\text { Circulating } \\
\text { levels }\end{array}$ & Brain/Pit expres. & $\begin{array}{l}\text { Periph. } \\
\text { expres. }\end{array}$ & $\begin{array}{l}\text { Circulating } \\
\text { levels }\end{array}$ & Brain/Pit expres. & $\begin{array}{l}\text { Periph. } \\
\text { expres. }\end{array}$ & $\begin{array}{l}\text { Circulating } \\
\text { levels }\end{array}$ & $\begin{array}{l}\text { Brain } \\
\text { expres. }\end{array}$ & $\begin{array}{l}\text { Periph. } \\
\text { expres. }\end{array}$ \\
\hline $\mathrm{GH}$ & $\begin{array}{l}\text { Chinook } \\
\text { salmon }=(182) \\
\text { Tilapia } \uparrow(97)\end{array}$ & & & $\begin{array}{l}\text { Channel } \\
\text { catfish }=\uparrow \\
(183) \\
\text { Chinook } \\
\text { salmon } \uparrow \\
(182) \\
\text { Coho salmon } \\
\uparrow(76,184) \\
\text { Fine flounder } \\
\uparrow(162) \\
\text { Rainbow trout } \\
\uparrow(185-187) \\
\text { Tilapia } \\
\uparrow=(97,188, \\
189)\end{array}$ & $\begin{array}{l}\text { Channel catfish }=\uparrow \\
\text { (183) } \\
\text { Cichlasoma } \\
\text { dimerus }=(190) \\
\text { Crucian carp } \downarrow \text { (191) } \\
\text { Grouper } \uparrow(192) \\
\text { Tilapia } \uparrow(188) \\
\end{array}$ & & & & \\
\hline |GF-I & $\begin{array}{l}\text { Chinook } \\
\text { salmon }=\downarrow \\
(182) \\
\text { Tilapia } \downarrow \text { (97) }\end{array}$ & & $\begin{array}{l}\text { Chinook } \\
\text { salmon }=\downarrow \\
\text { (182) }\end{array}$ & $\begin{array}{l}\text { Channel } \\
\text { catfish } \downarrow \text { (183) } \\
\text { Chinook } \\
\text { salmon } \downarrow \\
\text { (182) } \\
\text { Coho salmon } \\
\downarrow(184) \\
\text { Rainbow trout } \\
\downarrow(185) \\
\text { Tilapia } \downarrow \\
(97,188,189, \\
193)\end{array}$ & & $\begin{array}{l}\text { Atlantic } \\
\text { salmon }=(145)( \\
\text { Channel } \\
\text { catfish } \downarrow \text { (183) } \\
\text { Chinook } \\
\text { salmon } \downarrow \\
\text { (182) } \\
\text { Coho salmon } \\
\text { Cichlasoma } \\
\text { dimerus }=\downarrow \\
\text { (190) } \\
\downarrow=(76) \\
\text { Crucian carp } \\
\downarrow \text { (191) } \\
\text { Grouper } \downarrow \\
\text { (192) } \\
\text { Tilapia } \downarrow \text { (188, } \\
\text { 189, 193) } \\
\text { Yellowtail = (194) }\end{array}$ & $\begin{array}{l}\text { Brown trout } \downarrow \\
\text { (195) } \\
\text { Masu salmon } \\
\downarrow \text { (196) } \\
\text { 4) } \\
\text { 4 }\end{array}$ & & $\begin{array}{l}\text { Masu } \\
\text { salmon }=(196)\end{array}$ \\
\hline
\end{tabular}

$=$ levels not altered; $\uparrow$ levels upregulated; $\downarrow$ levels downregulated

high GH plasmatic levels together with low levels of IGF-I (and also insulin) induces lipolysis making fatty acids available to peripheral tissues (197). Using this mechanism, fish are capable to usually maintain its internal functions during fasting, avoiding somatic growth.

Studies on the effects of feed quantity are available regarding the GH-IGF system. In general, increased feed ration results in inverse changes in GH-IGF system hormones, compared to fasting $(182,198-200)$. This state in which plasmatic GH levels are low and IGFs are high is correlated with an increase in the somatic growth rate of fish (200). However, if feed ration size is considerable, plasmatic GH levels are high, and GHRs and IGFs levels remain low. This probably diminishes feed utilization for growth (199).

\section{Diet Composition $\mathrm{CHO}$}

Diet composition is another important factor regulating the GH-IGF system and somatic growth (197). Several works have been carried out in the past years aiming to determine the effect of dietary carbohydrates, especially glucose, on GH in fish. Rodgers et al. (201) demonstrated that when tilapia pituitaries were incubated in the presence of varying glucose concentrations, the quantity of $\mathrm{GH}$ released is inversely related to glucose concentration in the culture media. However, Riley et al. (202) found no changes in pituitary $\mathrm{GH}$ mRNA or plasma $\mathrm{GH}$ in response to intraperitoneal (IP) glucose injection. In the liver, IP glucose treatment significantly elevates the levels of GHSR mRNAs. Although the IGF-I mRNA expression was not 
altered by the IP glucose injection, the IGF-I plasma levels were significantly reduced in tilapia (202). In an in vitro assay carried out by our group (203), it was found that incubation of goldfish hepatopancreas with different glucose concentrations significantly increases the expression of $g h r-I, g h r-I I$, igf-I, and igf-II mRNAs at $4 \mathrm{~h}$. The increase in GHR and GH mRNAs caused by glucose could be related its insulinotropic effect, as such outcomes were demonstrated in mammals (204). IGFs were postulated as regulators of glucose uptake in fish (205), and in mammals (206), likely through the modulation of GLUT1 glucose transporter (207). GH has been shown to have a hyperglycemic effect in several species of fish, and it is glycogenolytic, glycolytic and gluconeogenetic in some tissues, including the liver, brain, and gill (208). Therefore, IGFs seem to play a role in maintaining the balance between $\mathrm{GH}$ and insulin to promote normal carbohydrate metabolism.

\section{Proteins}

Dietary protein seems to regulate the hepatic IGF-I expression and secretion, as it was demonstrated in mirror carp (209) and Nile tilapia juveniles (210). In both cases, authors found a significant correlation between dietary protein levels and hepatic IGF-I mRNA expression. Moreover, Qiang et al. (210) found that the increase in the dietary protein content not only increases the IGF-I mRNA expression but also increases the plasmatic IGFI levels. These authors also reported a significant correlation between the fish-specific growth rate (SGR) and the IGF-I plasmatic levels and/or IGF-I hepatic mRNA expression. In gibel carp (211), it was found that an increase in dietary protein levels leads to an increase in SGR and hepatic IGF-I mRNA levels, although a decrease in both parameters was observed with extremely high protein levels in the diet. Moreover, Tu et al. (211) reported that GH mRNA expression in pituitary shows the opposite trend compared to hepatic IGF-I mRNA. PérezSánchez et al. (199) found that gilthead seabream fed on low protein diets had significantly higher $\mathrm{GH}$, but lower hepatic $\mathrm{GH}$ binding and lower IGF-I levels than fish fed on higher protein diets. This situation resembles the one discussed previously in which fish under fasting shows diminished IGFs production and elevated GH. Therefore, low and very high dietary protein levels may influence the GH-IGF axis through the same mechanism as fasting, possibly by direct control of pituitary hormone secretion by circulating nutrients.

\section{Lipids}

Dietary lipids have also been shown to modulate fish growth. In several fish species, an increase in the dietary lipid content or a decrease in the protein/lipid ratio was shown to have a negative effect on growth, as reported for the Senegalese sole (212), the turbot (213), and the flounder (214). Although a few studies show the effect of dietary lipids on fish growth performance, there is little knowledge on their effect on the regulation of the GH-IGF system. In Senegalese sole, it was demonstrated that an increase in dietary lipid content increases the hepatic IGF-I mRNA expression, and this was inversely correlated with the somatic growth (215). In pejerrey, an increase from 10 to $21 \%$ in the dietary lipid amount generates a decrease in the hepatopancreatic
GHR-II mRNA expression, while no changes in somatic growth were found (216). In largemouth bass, the effects of different carbohydrates/lipids (CHO/L) ratios on the GH-IGF system, as well as on the somatic growth, were studied. Authors found that $\mathrm{CHO} / \mathrm{L}$ ratios from 0.32 to 2.36 significantly upregulate $\mathrm{GH}$ mRNA expression and downregulate IGF-I mRNA expression. Higher ratios did not exert any further effects on them. A positive correlation between hepatic IGF-I mRNA levels and specific growth rates with varying dietary $\mathrm{CHO} / \mathrm{LIP}$ ratios was found (217). All these findings indicate that dietary lipid level can differentially regulate the growth endocrine axis, at least at the transcription level. That can be directly associated with the role of these hormones in regulating lipid homeostasis, and particularly with the direct lipolytic effects of GH and the promotion of tissue growth by IGF-I. Moreover, results presented here also indicate that hepatic IGF-I mRNA as well as its plasma abundance could be a reliable index to assess growth and nutritional fitness.

Several studies have been focused on the effect of fatty acids present in dietary lipids and in the replacement of dietary fish oil by vegetable oil on fish growth. In most of the cases, an increase in the dietary amount of fatty acids from vegetable sources causes an increase in GH mRNA expression and/or circulating levels, while diminishes the IGF-I plasmatic and GHR-I mRNA levels (179, 203, 218). The hepatic mRNA expression of GHR-II and IGF-II seems to be constitutive and not affected by dietary fatty acids in sea bream (218), while in pejerrey the replacement of dietary fish oil by sunflower oil increases their mRNA expression (179). As a general conclusion, large replacements of high-unsaturated fatty acids by low-unsaturated fatty acids in fish diet lead to the GH-IGF system pattern to resemble one observed during fasting.

\section{Micronutrients}

The effect of micronutrients on the expression of GH-IGF system components was not extensively studied. To the best of our knowledge there is only one work reporting an effect of vitamins on igf-I expression (219). In this, the authors found that $4 \%$ of dietary vitamins generate an increase in the IGF-I mRNA expression in sea bass larvae after 38 days of the experiment, compared with diets containing lower or higher amounts.

\section{FUTURE PERSPECTIVES}

The role of nutrition as a potent modulator of the endocrine system governing feeding behavior and growth in fish is described in this review. Aquaculture research is a topic of increasing interest due to the demand for sustainable food production. That makes it essential to summarize the knowledge generated in the last past years in order to identify the weak points and to determine the direction in which new studies should be focused. As described here, food availability and feed composition are two of the most influent external signals modulating appetite-regulating and growth modulatory hormones. Future research should, therefore, be focused on tapping into this knowledge, expanding it in depth, and exploring its use to enhance feeding and growth in fish. Such approaches will eventually lead to increased yield in cultured fish. 
It is also interesting to note the species-specific differences in the effect of feeding on the expression of appetite-regulating hormones and the GH-IGF system. Such differences could be related to the feeding behavior of specific fish species, as the carnivorous, herbivorous and omnivorous fish tend to fall into three different groups of responses. This fact also reflects the flexibility of metabolic systems, considering that the same components present in all fish species could generate distinct responses from the same food stimuli. Therefore, it is critical to bear in mind that results obtained from one species of fish might not apply to another.

As a final consideration, it would be interesting to broaden our knowledge of the crosstalk between hormones regulating food intake and those regulating growth. For instance, $\mathrm{GH}$ and IGF-I not only regulate somatic growth but can at the same time modulate lipid and carbohydrate metabolism, respectively (220). Others, including ghrelin, could stimulate food intake (221), and also GH release from pituitary $(222,223)$. Likewise, it has been shown that leptin directly regulates the expression of IGFs and GHRs in fish hepatopancreas (224). These are a few examples of the crosslinks mentioned above, that serve to highlight the close interaction that exists between hormones controlling food intake and growth to ensure the proper growth and development of fish. Thus, comprehensive approaches to determine both metabolic and growth regulatory hormone

\section{REFERENCES}

1. Douros JD, Baltzegar DA, Mankiewicz J, Taylor J, Yamaguchi Y, Lerner DT, et al. Control of leptin by metabolic state and its regulatory interactions with pituitary growth hormone and hepatic growth hormone receptors and insulin like growth factors in the tilapia (Oreochromis mossambicus). Gen Comp Endocrinol. (2017) 240:227-37. doi: 10.1016/j.ygcen.2016. 07.017

2. Mommsen TP. Paradigms of growth in fish. Comp Biochem Physiol B Biochem Mol Biol. (2001) 129:207-19. doi: 10.1016/S1096-4959(01) 00312-8

3. Näslund E, Hellström PM. Appetite signaling: From gut peptides and enteric nerves to brain. Physiol Behav. (2007) 92:256-62. doi: 10.1016/j.physbeh.2007.05.017

4. Reindl KM, Sheridan MA. Peripheral regulation of the growth hormoneinsulin-like growth factor system in fish and other vertebrates. Comp Biochem Physiol A Mol Integr Physiol. (2012) 163:231-45. doi: 10.1016/j.cbpa.2012.08.003

5. Kageyama H, Takenoya F, Shiba K, Shioda S. Neuronal circuits involving ghrelin in the hypothalamus-mediated regulation of feeding. Neuropeptides. (2010) 44:133-8. doi: 10.1016/j.npep.2009.11.010

6. Crespo CS, Cachero AP, Jiménez LP, Barrios V, Ferreiro EA. Peptides and food intake. Front Endocrinol. (2014) 5:58. doi: 10.3389/fendo.2014. 00058

7. Michel M, Volkoff H, Conde-Sieira M, Soengas JL. Nutrient sensing systems in fish: impact on food intake regulation and energy homeostasis. Nutr Sens Mech Fish. (2017) 10:1-21. doi: 10.3389/fnins.2016.00603

8. Efeyan A, Comb WC, Sabatini DM. Nutrient-sensing mechanisms and pathways. Nature. (2015) 517:302-10. doi: 10.1038/nature14190

9. Gatlin DM. Food intake in fish. In: Houlihan D, Boujard T, Jobling M, editors. COST Action 827. Oxford, UK: Blackwell Science (2001). p. 259. doi: 10.1016/S0044-8486(02)00066-2

10. Council NR. Nutrient Requirements of Horses. 6th ed. Washington, DC: The National Academies Press (2007).

11. Mommsen TP. (2002). Carbohydrates in fish nutrition effects on growth, glucose metabolism and hepatic enzymes. responses to nutritional challenges are more desirable from an aquaculture perspective. Future research must focus to identify gaps in knowledge, including the ones identified above. In addition, the use of alternate feed ingredients, use of endogenous feeding and growth regulatory factors as feed additives, and employing hormones using targeted molecular and cellular approaches should be explored to modulate growth rate and yield in cultured species.

\section{AUTHOR CONTRIBUTIONS}

JB prepared larger sections and complied the manuscript. $\mathrm{AB}$, LS, JR, and CV wrote smaller sections. SU provided the idea, outline, provided feedback, and corrected the manuscript.

\section{ACKNOWLEDGMENTS}

The research on fish metabolism in the laboratory of SU has been supported by Discovery Grants from the Natural Sciences and Engineering Research Council (NSERC) of Canada. Research lab infrastructure was purchased using a Leaders Opportunities Fund from the Canada Foundation for Innovation (CFI), and an Establishment Grant from the Saskatchewan Health Research Foundation (SHRF).

12. Polakof S, Panserat S, Soengas JL, Moon TW. Glucose metabolism in fish: a review. J Comp Physiol B Biochem Syst Environ Physiol. (2012) 182:1015-45. doi: 10.1007/s00360-012-0658-7

13. Moon TW. Glucose intolerance in teleost fish: fact or fiction? Comp Biochem Physiol B Biochem Mol Biol. (2001) 129:243-9. doi: 10.1016/S1096-4959(01)00316-5

14. Soengas JL, Aldegunde M. Energy metabolism of fish brain. Comp Biochem Physiol B Biochem Mol Biol. (2002) 131:271-96. doi: 10.1016/S1096-4959(02)00022-2

15. Sargent J, Tocher D, Bell J. The lipids. Fish Nutr. (2002) 181-257. doi: 10.1016/B978-012319652-1/50005-7

16. Torstensen BE, Lie $\emptyset$, Frøyland L. Lipid metabolism and tissue composition in Atlantic salmon (Salmo salar L.)- Effects of capelin oil, palm oil, and oleic scid-enriched sunflower oil as dietary lipid sources. Lipids. (2000) 35:653-64. doi: 10.1007/s11745-000-0570-6

17. Tocher DR, Fraser AJJ, Sargent JRR, Gamble JCC. Fatty acid composition of phospholipids and neutral lipids during embryonic and early larval development in atlantic herring (Clupea harengus, L.). Lipids. (1985) 20:6974. doi: 10.1007/BF02534210

18. Sheridan MA. Regulation of Lipid-Metabolism in Poikilothermic Vertebrates. Comp Biochem Physiol B Biochemistry Mol Biol. (1994) 107:495-508. doi: 10.1016/0305-0491(94)90176-7

19. Taylor P, Tocher DR. Reviews in fisheries science metabolism and functions of lipids and fatty acids in teleost fish. Rev Fish Sci. (2010) 37-41. doi: 10.1080/713610925

20. Craig S, Helfrich LA. Understanding Fish Nutrition Feeds, and Feeding. Virginia Coop Ext (2002) p. 1-18. Available online at: https://pubs.ext.vt.edu/ 420/420-256/420-256.html

21. Huntingford F, Jobling M, Kadri S. Aquaculture and Behavior. Chichester, UK: John Wiley \& Sons (2011).

22. Langhans W. Appetite regulation. In: Lobley GE, White A, MacRae JC, editors. Protein Metabolism and Nutrition. Wageningen Pers (1999). p. $225-51$.

23. Lawrence JM. Preliminary results on the use of potassium permanganate to counteract the effects of rotenone on fish. Progress Fish-Culturist. (1956) $18: 15-21$. 
24. Chantranupong L, Wolfson RL, Sabatini DM. Nutrient-sensing mechanisms across evolution. Cell. (2015) 161:67-83. doi: 10.1016/j.cell.2015.02.041

25. Morgan K, Obici S, Rossetti L. Hypothalamic responses to long-chain fatty acids are nutritionally regulated. J Biol Chem. (2004) 279:31139-48. doi: 10.1074/jbc.M400458200

26. Pocai A, Obici S, Schwartz GJ, Rossetti L. A brain-liver circuit regulates glucose homeostasis. Cell Metab. (2005) 1:53-61. doi: 10.1016/j.cmet.2004.11.001

27. Roh E, Song DK, Kim, M-S. Emerging role of the brain in the homeostatic regulation of energy and glucose metabolism. Exp Mol Med. (2016) 48:e216. doi: 10.1038/emm.2016.4

28. Morton GJ, Meek TH, Schwartz MW. Neurobiology of food intake in health and disease. Nat Rev Neurosci. (2014) 15:367-78. doi: 10.1038/nrn3745

29. Rogers RC, Ritter S, Hermann GE, Rc R, Ritter S, Hindbrain HGE. Hindbrain cytoglucopenia-induced increases in systemic blood glucose levels by 2-deoxyglucose depend on intact astrocytes and adenosine release. Am J Physiol Regul Integr Comp Physiol. (2016) 310:1102-8. doi: 10.1152/ajpregu.00493.2015

30. Lerch M, Mayrhofer M, Zerbe O. Structural similarities of micelle-bound peptide YY (PYY) and neuropeptide Y (NPY) are related to their affinity profiles at the $\mathrm{Y}$ receptors. $J$ Mol Biol. (2004) 339:1153-1168. doi: 10.1016/j.jmb.2004. 04.032

31. Cerdá-Reverter JM, Larhammar D. Neuropeptide Y family of peptides: structure, anatomical expression, function, and molecular evolution. Biochem Cell Biol. (2000) 78:371-92. doi: 10.1139/o00-004

32. Campos VF, Collares T, Deschamps JC, Seixas FK, Dellagostin OA, Lanes CF, et al. Identification, tissue distribution and evaluation of brain neuropeptide Y gene expression in the Brazilian flounder Paralichthys orbignyanus. J Biosci. (2010) 35:405-13. doi: 10.1007/s12038-010-0046-y

33. Volkoff $H$. The neuroendocrine regulation of food intake in fish: a review of current knowledge. Front Neurosci. (2016) 10:540. doi: 10.3389/fnins.2016.00540

34. Cerdá-Reverter JM, Peter RE. Endogenous melanocortin antagonist in fish: structure, brain mapping, and regulation by fasting of the goldfish agouti-related protein gene. Endocrinology. (2003) 144:4552-61. doi: 10.1210/en.2003-0453

35. Cerdá-Reverter JM, Canosa LF. Neuroendocrine systems of the fish brain. In: Bernier NJ, Van Der Kraak G, Farrell AP, Brauner CJ, editors. Fish Neuroendocrinology: Fish Physiology. (2009) 28, p. 3-74.

36. Noon LA, Franklin JM, King PJ, Goulding NJ, Hunyady L, Clark AJ. Failed export of the adrenocorticotrophin receptor from the endoplasmic reticulum in non-adrenal cells: evidence in support of a requirement for a specific adrenal accessory factor. J Endocrinol. (2002) 174:17-25. doi: $10.1677 /$ joe. 0.1740017

37. Metherell LA, Chapple JP, Cooray S, David A, Becker C, Rüschendorf F, et al. Mutations in MRAP, encoding a new interacting partner of the ACTH receptor, cause familial glucocorticoid deficiency type 2. Nat Genet. (2005) 37:166-70. doi: 10.1038/ng1501

38. Douglass J, McKinzie AA, Couceyro P. PCR differential display identifies a rat brain mRNA that is transcriptionally regulated by cocaine and amphetamine. J Neurosci. (1995) (3 Pt 2):2471-81. doi: 10.1523/JNEUROSCI.15-03-02471.1995

39. Volkoff H, Peter RE. Characterization of two forms of cocaine- and amphetamine-regulated transcript (CART) peptide precursors in goldfish: molecular cloning and distribution, modulation of expression by nutritional status, and interactions with leptin. Endocrinology. (2001) 2:5076-88. doi: 10.1210/endo.142.12.8519

40. Shewale SA, Gaupale TC, Bhargava S. Temperature dependent changes in cocaine-and amphetamine regulated transcript (CART) peptide in the brain of tadpole, Sylvirana temporalis. Gen Comp Endocrinol. (2015) 220:61-9. doi: 10.1016/j.ygcen.2014.06.025

41. Gilon P. Cocaine-and amphetamine-regulated transcript: a novel regulator of energy homeostasis expressed in a subpopulation of pancreatic islet cells. Diabetologia. (2016) 59:1855-9. doi: 10.1007/s00125-016-4052-y

42. Ferguson AV, Samson WK. The orexin/hypocretin system: a critical regulator of neuroendocrine and autonomic function. Front Neuroendocrinol. (2003) 24:141-50. doi: 10.1016/S0091-3022(03)00028-1
43. Rodgers RJ, Ishii Y, Halford JC, Blundell JE. Orexins and appetite regulation. Neuropeptides. (2002) 36:303-25 doi: 10.1016/S0143-4179(02)00085-9

44. Hagan JJ, Leslie RA, Patel S, Evans ML, Wattam TA, Holmes S, et al. Orexin A activates locus coeruleus cell firing and increases arousal in the rat. Proc Natl Acad Sci USA. (1999) 96:10911-10916. doi: 10.1073/pnas.96.19.10911

45. Volkoff H, Peter RE. Interactions between orexin A, NPY and galanin in the control of food intake of the goldfish, Carassius auratus. Regul Pept. (2001) 101:59-72. doi: 10.1016/S0167-0115(01)00261-0

46. Kawauchi H, Kawazoe I, Tsubokawa M, Kishida M, Baker BI. Characterization of melanin-concentrating hormone in chum salmon pituitaries. Nature. (1983) 305:321-3. doi: 10.1038/305321a0

47. Logan DW, Bryson-Richardson RJ, Pagán KE, Taylor MS, Currie PD, Jackson IJ. The structure and evolution of the melanocortin and $\mathrm{MCH}$ receptors in fish and mammals. Genomics. (2003) 81:184-91. doi: 10.1016/S0888-7543(02)00037-X

48. Oh,-I. S., Shimizu H, Satoh T, Okada S, Sachika A, Inoue K, et al. Identification of nesfatin-1 as a satiety molecule in the hypothalamus. Nature. (2006) 443:709-12. doi: 10.1038/nature05162

49. Iwasaki Y, Nakabayashi H, Kakei M, Shimizu H, Mori M, Yada T. Nesfatin1 evokes $\mathrm{Ca} 2+$ signaling in isolated vagal afferent neurons via $\mathrm{Ca} 2+$ influx through N-type channels. Biochem Biophys Res Commun. (2009) 390:958-62. doi: 10.1016/j.bbrc.2009.10.085

50. Gonzalez R, Kerbel B, Chun A, Unniappan S. Molecular, cellular and physiological evidences for the anorexigenic actions of nesfatin-1 in goldfish. PLoS ONE. (2010) 5:e15201. doi: 10.1371/journal.pone.0015201

51. Lin F, Zhou C, Chen H, Wu H, Xin Z, Liu J, et al. Molecular characterization, tissue distribution and feeding related changes of NUCB2A/nesfatin-1 in Ya-fish (Schizothorax prenanti). Gene (2014) 536:238-46. doi: 10.1016/j.gene.2013.12.031

52. Caldwell LK, Pierce AL, Riley LG3m Duncan CA, Nagler JJ. Plasma nesfatin-1 is not affected by long-term food restriction and does not predict rematuration among iteroparous female rainbow trout (Oncorhynchus mykiss). PLoS ONE. (2014) 9:e85700. doi: 10.1371/journal.pone.00 85700

53. Kojima M, Hosoda H, Date Y, Nakazato M, Matsuo H, Kangawa K, et al. Ghrelin is a growth-hormone-releasing acylated peptide from stomach. Nature. (1999) 402:656-60. doi: 10.1038/45230

54. Kaiya H, Kojima M, Hosoda H, Moriyama S, Takahashi A, Kawauchi H, et al. Peptide purification, complementary deoxyribonucleic acid (DNA) and genomic DNA cloning, and functional characterization of ghrelin in rainbow trout. Endocrinology. (2003) 144:5215-26. doi: 10.1210/en. 2003-1085

55. Kaiya H, Miyazato M, Kangawa K, Peter RE, Unniappan S. Ghrelin: a multifunctional hormone in non-mammalian vertebrates. Comp Biochem Physiol A Mol Integr Physiol. (2008) 149:109-28. doi: 10.1016/j.cbpa.2007.12.004

56. Jönsson E. The role of ghrelin in energy balance regulation in fish. Gen Comp Endocrinol. (2013) 187:79-85. doi: 10.1016/j.ygcen.2013.03.013

57. Salmerón C, Johansson M, Asaad M, Angotzi AR, Rønnestad I, Stefansson $\mathrm{SO}$, et al. Roles of leptin and ghrelin in adipogenesis and lipid metabolism of rainbow trout adipocytes in vitro. Comp Biochem Physiol A Mol Integr Physiol. (2015) 188:40-8. doi: 10.1016/j.cbpa.2015.06.017

58. Beinfeld MC. Biosynthesis and processing of pro CCK: recent progress and future challenges. Life Sci. (2003) 72:747-57. doi: 10.1016/S0024-3205(02)02330-5

59. Kurokawa T, Suzuki T, Hashimoto H. Identification of gastrin and multiple cholecystokinin genes in teleost. Peptides. (2003) 24:227-35. doi: 10.1016/S0196-9781(03)00034-2

60. Aldman G, Holmgren S. Intraduodenal fat and amino acids activate gallbladder motility in the rainbow trout, Oncorhynchus mykiss. Gen Comp Endocrinol. (1995) 100:27-32. doi: 10.1006/gcen.1995.1128

61. Himick BA, Peter RE. CCK/gastrin-like immunoreactivity in brain and gut, and CCK suppression of feeding in goldfish. Am J Physiol. (1994) 267(3 Pt 2):R841-51.

62. Volkoff H, Eykelbosh AJ, Peter RE. Role of leptin in the control of feeding of goldfish Carassius auratus: interactions with cholecystokinin, neuropeptide Y and orexin A, and modulation by fasting. Brain Res. (2003) 972:90-109. doi: 10.1016/S0006-8993(03)02507-1 
63. Gonzalez R, Unniappan S. Molecular characterization, appetite regulatory effects and feeding related changes of peptide YY in goldfish. Gen Comp Endocrinol. (2010) 166:273-9. doi: 10.1016/j.ygcen.2009.09.008

64. Batterham RL, Cowley MA, Small CJ, Herzog H, Cohen MA, Dakin CL, et al. Gut hormone PYY(3-36) physiologically inhibits food intake. Nature. (2002) 418:650-4. doi: 10.1038/nature00887

65. Cerdá-Reverter JM, Martínez-Rodríguez G, Zanuy S, Carrillo M, Larhammar D. Molecular evolution of the neuropeptide Y (NPY) family of peptides: cloning of three NPY-related peptides from the sea bass (Dicentrarchus labrax). Regul Pept. (2000) 95:25-34. doi: 10.1016/S0167-0115(00)00132-4

66. Plisetskaya EM, Mommsen TP. Glucagon and glucagonlike peptides in fishes. Int Rev Cytol. (1996) 168:187-257. doi: 10.1016/S0074-7696(08)60885-2

67. Yeung CM, Mojsov S, Mok PY, Chow BK. Isolation and structurefunction studies of a glucagon-like peptide 1 receptor from goldfish Carassius auratus: identification of three charged residues in extracellular domains critical for receptor function. Endocrinology. (2002) 143:4646-54. doi: 10.1210/en.2002-220694

68. Londraville RL, Macotela Y, Duff RJ, Easterling MR, Liu Q, Crespi EJ. Comparative endocrinology of leptin: assessing function in a phylogenetic context. Gen Comp Endocrinol. (2014) 203:146-57. doi: 10.1016/j.ygcen.2014.02.002

69. Song YF, Wu K, Tan XY, Zhang LH, Zhuo MQ, Pan YX, et al. Effects of recombinant human leptin administration on hepatic lipid metabolism in yellow catfish Pelteobagrus fulvidraco: in vivo and in vitro studies. Gen Comp Endocrinol. (2015) 212:92-9. doi: 10.1016/j.ygcen.2015.01.022

70. Gong N, Jönsson E, Björnsson BT. Acute anorexigenic action of leptin in rainbow trout is mediated by the hypothalamic Pi3k pathway. J Mol Endocrinol. (2016) 56:227-38. doi: 10.1530/JME-15-0279

71. Musumeci G, Trovato FM, Avola R, Imbesi R, Castrogiovanni P. Serotonin/growth hormone/insulin-like growth factors axis on pre- and post-natal development: a contemporary review. OA Anat. (2013) 1:1-7.

72. Johnsson JI, Björnsson BT. Growth hormone increases growth rate, appetite and dominance in juvenile rainbow trout, Oncorhynchus mykiss. Anim. Behav. (1994) 48:177-86. doi: 10.1006/anbe.1994.1224

73. Abrahams MV, Sutterlin A. The foraging and antipredator behaviour of growth-enhanced transgenic Atlantic salmon. Anim Behav. (1999) 58:93342. doi: 10.1006/anbe.1999.1229

74. Reinecke M, Björnsson BT, Dickhoff WW, McCormick SD, Navarro I, Power DM, et al. Growth hormone and insulin-like growth factors in fish: where we are and where to go. Gen Comp Endocrinol. (2005) 142:20-4. doi: 10.1016/j.ygcen.2005.01.016

75. Pérez-Sánchez J, Calduch-Giner JA, Mingarro M, de Celis SVR, GomezRequeni P, Saera-Vila A, et al. Overview of fish growth hormone family. New insights in genomic organization and heterogeneity of growth hormone receptors. Fish Physiol Biochem. (2002) 27:243-58. doi: 10.1023/B:FISH.0000032729.72746.c8

76. Duan C, Plisetskaya EM. Nutritional regulation of insulin-like growth factorI mRNA expression in salmon tissues. J Endocrinol. (1993) 139:243-52. doi: $10.1677 /$ joe. 0.1390243

77. Duguay SJ, Park LK, Samadpour M, Dickhoff WW. Nucleotide sequence and tissue distribution of three insulin-like growth factor I prohormones in salmon. Mol Endocrinol. (1992) 6:1202-10. doi: 10.1210/mend.6.8.1406698

78. Shamblott MJ, Chen TT. Identification of a second insulin-like growth factor in a fish species. Proc Natl Acad Sci USA. (1992) 89 8913-7. doi: $10.1073 /$ pnas.89.19.8913

79. Perrot V, Moiseeva EB, Gozes Y, Chan SJ, Funkenstein B. Insulin-like growth factor receptors and their ligands in gonads of a hermaphroditic species, the gilthead seabream (Sparus aurata): expression and cellular localization. Biol Reprod. (2000) 63:229-41. doi: 10.1095/biolreprod63.1.229

80. Reinecke M, Collet C. The phylogeny of the insulin-like growth factors. Int Rev Cytol. (1998) 183:1-94. doi: 10.1016/S0074-7696(08)60142-4

81. Codina M, Castillo J, Gutiérrez J, Navarro I. Role of IGF-II in the metabolism and proliferation of rainbow trout muscle cells. Gen Comp Endocrinol. (2004) (Abstract 5th ISFE, O41).

82. Shamblott M, Cheng C, Bolt D, Chen T. Appearance of insulin-like growth factor mRNA in the liver and pyloric ceca of a teleost in response to exogenous growth hormone. Proc Natl Acad Sci USA. (1995) 92:6943-6. doi: 10.1073/pnas.92.15.6943

83. Tse MCL, Vong QP, Cheng CHK, Chan KM. PCR-cloning and gene expression studies in common carp (Cyprinus carpio) insulinlike growth factor-II. Biochim Biophys Acta. (2002) 1575:63-74. doi: 10.1016/S0167-4781(02)00244-0

84. Vong QP, Chan KM, Cheng CHK. Quantification of common carp (Cyprinus carpio) IGF-I and IGF-II mRNA by real-time PCR: differential regulation of expression by GH. J Endocrinol. (2003) 178:513-21. doi: $10.1677 /$ joe. 0.1780513

85. Campos VF, Robaldo RB, Deschamps JC, Seixas FK, McBride AJA, Marins LF, et al. Neuropeptide Y gene expression around meal time in the Brazilian flounder Paralichthys orbignyanus. J Biosci. (2012) 37:227-32. doi: 10.1007/s12038-012-9205-7

86. Nguyen MV, Jordal A-EO, Espe M, Buttle L, Lai HV, Rønnestad I. Feed intake and brain neuropeptide Y (NPY) and cholecystokinin (CCK) gene expression in juvenile cobia fed plant-based protein diets with different lysine to arginine ratios. Comp Biochem Physiol A Mol Integr Physiol. (2013) 165:328-37. doi: 10.1016/j.cbpa.2013.04.004

87. Volkoff H, Sabioni RE, Cyrino JEP. Appetite regulating factors in dourado, Salminus brasiliensis: cDNA cloning and effects of fasting and feeding on gene expression. Gen Comp Endocrinol. (2016) 237:34-42. doi: 10.1016/j.ygcen.2016.07.022

88. Volkoff H, Estevan Sabioni R, Coutinho LL, Cyrino JEP. Appetite regulating factors in pacu (Piaractus mesopotamicus): tissue distribution and effects of food quantity and quality on gene expression. Comp Biochem Physiol A Mol Integr Physiol. (2017) 203:241-54. doi: 10.1016/j.cbpa.2016.09.022

89. Wall A, Volkoff H. Effects of fasting and feeding on the brain mRNA expressions of orexin, tyrosine hydroxylase (TH), PYY and CCK in the Mexican blind cavefish (Astyanax fasciatus mexicanus). Gen Comp Endocrinol. (2013) 183:44-52. doi: 10.1016/j.ygcen.2012.12.011

90. Xu M, Volkoff H. Molecular characterization of prepro-orexin in Atlantic cod (Gadus morhua): cloning, localization, developmental profile and role in food intake regulation. Mol Cell Endocrinol. (2007) 271:28-37. doi: 10.1016/j.mce.2007.03.003

91. Yuan D, Wei R, Wang T, Wu Y, Lin F, Chen H, et al. Appetite regulation in Schizothorax prenanti by three CART genes. Gen Comp Endocrinol. (2015) 224:194-204. doi: 10.1016/j.ygcen.2015.08.015

92. Zhang X, Gao Y, Tang N, Qi J, Wu Y, Hao J, et al. One evidence of cocaineand amphetamine-regulated transcript (CART) has the bidirectional effects on appetite in Siberian sturgeon (Acipenser baerii). Fish Physiol Biochem. (2018) 44:411-22. doi: 10.1007/s10695-017-0444-2

93. Hatef A, Shajan S, Unniappan S. Nutrient status modulates the expression of nesfatin-1 encoding nucleobindin 2A and 2B mRNAs in zebrafish gut, liver and brain. Gen Comp Endocrinol. (2015) 215:51-60. doi: 10.1016/j.ygcen.2014.09.009

94. Blanco AM, Gómez-Boronat M, Redondo I, Valenciano AI, Delgado MJ. Periprandial changes and effects of short- and long-term fasting on ghrelin, GOAT, and ghrelin receptors in goldfish (Carassius auratus). J. Comp. Physiol B. (2016) 186:727-38. doi: 10.1007/s00360-0160986-0

95. Unniappan S, Canosa LF, Peter RE. Orexigenic actions of ghrelin in goldfish: feeding-induced changes in brain and gut mRNA expression and serum levels, and responses to central and peripheral injections. Neuroendocrinology. (2004) 79:100-8. doi: 10.1159/000076634

96. Jönsson E, Forsman A, Einarsdottir IE, Kaiya H, Ruohonen K, Björnsson BT. Plasma ghrelin levels in rainbow trout in response to fasting, feeding and food composition, and effects of ghrelin on voluntary food intake. Comp Biochem Physiol A Mol Integr Physiol. (2007) 147:1116-24. doi: 10.1016/j.cbpa.2007.03.024

97. Fox BK, Breves JP, Hirano T, Grau EG. Effects of short- and long-term fasting on plasma and stomach ghrelin, and the growth hormone/insulinlike growth factor I axis in the tilapia, Oreochromis mossambicus. Domest Anim Endocrinol. (2009) 37:1-11. doi: 10.1016/j.domaniend.2009.01.001

98. Zhou C, Zheng J, Lei L, Yuan D, Zhu C, Ye H, et al. Evidence that ghrelin may be associated with the food intake of gibel carp (Carassius auratus gibelio). Fish Physiol. Biochem. (2016) 42:1637-46. doi: 10.1007/s10695-016-0246-y 
99. Peddu SC, Breves JP, Kaiya H, Gordon Grau E, Riley LG. Pre- and postprandial effects on ghrelin signaling in the brain and on the GH/IGFI axis in the Mozambique tilapia (Oreochromis mossambicus). Gen Comp Endocrinol. (2009) 161:412-8. doi: 10.1016/j.ygcen.2009.02.008

100. Wei R, Liu T, Zhou C, Zhang X, Yuan D, Wang T, et al. Identification, tissue distribution and regulation of preproghrelin in the brain and gut of Schizothorax prenanti. Regul Pept. (2013) 186:18-25. doi: 10.1016/j.regpep.2013.07.002

101. Hatef A, Yufa R, Unniappan S. Ghrelin O-Acyl Transferase in Zebrafish is an evolutionarily conserved peptide upregulated during calorie restriction. Zebrafish. (2015) 12:327-38. doi: 10.1089/zeb.2014.1062

102. Yuan D, Wang $\mathrm{T}$, Zhou $\mathrm{C}$, Lin $\mathrm{F}$, Chen $\mathrm{H}, \mathrm{Wu} \mathrm{H}$, et al. Leptin and cholecystokinin in Schizothorax prenanti: molecular cloning, tissue expression, and mRNA expression responses to periprandial changes and fasting. Gen Comp Endocrinol. (2014) 204:13-24. doi: 10.1016/j.ygcen.2014.05.013

103. Yuan D, Zhou C, Wang T, Lin F, Chen H, Wu H, et al. Molecular characterization and tissue expression of peptide YY in Schizothorax prenanti: effects of periprandial changes and fasting on expression in the hypothalamus. Regul Pept. (2014) 190-1:32-8. doi: 10.1016/j.regpep.2014.03.004

104. Chen H, Zhang X, Hao J, Chen D, Liu J, Gao Y, et al. Molecular cloning, expression analysis, and appetite regulatory effect of peptide YY in Siberian sturgeon (Acipenser baerii). Gene (2015) 563:172-9. doi: 10.1016/j.gene.2015.03.028

105. Yuan X, Li A, Liang X-F, Huang W, Song Y, He S, et al. Leptin expression in mandarin fish Siniperca chuatsi (Basilewsky): regulation by postprandial and short-term fasting treatment. Comp Biochem Physiol A Mol Integr Physiol. (2016) 194:8-18. doi: 10.1016/j.cbpa.2016.01.014

106. Tinoco AB, Nisembaum LG, Isorna E, Delgado MJ, de Pedro N. Leptins and leptin receptor expression in the goldfish (Carassius auratus). Regulation by food intake and fasting/overfeeding conditions. Peptides. (2012) 34:329-35. doi: 10.1016/j.peptides.2012.02.001

107. Moen A-GG, Finn RN. Short-term, but not long-term feed restriction causes differential expression of leptins in Atlantic salmon. Gen Comp Endocrinol. (2013) 183:83-8. doi: 10.1016/j.ygcen.2012.09.027

108. Murashita K, Kurokawa T, Ebbesson LOE, Stefansson SO, Rønnestad I. Characterization, tissue distribution, and regulation of agouti-related protein (AgRP), cocaine- and amphetamine-regulated transcript (CART) and neuropeptide Y (NPY) in Atlantic salmon (Salmo salar). Gen Comp Endocrinol. (2009) 162:160-71. doi: 10.1016/j.ygcen.2009.03.015

109. Ji W, Ping H-C, Wei K-J, Zhang G-R, Shi Z-C, Yang R-B, et al. Ghrelin, neuropeptide Y (NPY) and cholecystokinin (CCK) in blunt snout bream (Megalobrama amblycephala): cDNA cloning, tissue distribution and mRNA expression changes responding to fasting and refeeding. Gen Comp Endocrinol. (2015) 223:108-19. doi: 10.1016/j.ygcen.2015.08.009

110. Narnaware YK, Peter RE. Effects of food deprivation and refeeding on neuropeptide Y (NPY) mRNA levels in goldfish. Comp Biochem Physiol B Biochem Mol Biol. (2001) 129:633-7. doi: 10.1016/S1096-4959(01) 00359-1

111. Silverstein JT, Breininger J, Baskin DG, Plisetskaya EM. Neuropeptide Ylike gene expression in the salmon brain increases with fasting. Gen Comp Endocrinol. (1998) 110:157-65. doi: 10.1006/gcen.1998.7058

112. Babichuk NA, Volkoff H. Changes in expression of appetite-regulating hormones in the cunner (Tautogolabrus adspersus) during shortterm fasting and winter torpor. Physiol Behav. (2013) 120:54-63. doi: 10.1016/j.physbeh.2013.06.022

113. Yan P, Jia J, Yang G, Wang D, Sun C, Li W. Duplication of neuropeptide $\mathrm{Y}$ and peptide YY in Nile tilapia Oreochromis niloticus and their roles in food intake regulation. Peptides. (2017) 88:97-105. doi: 10.1016/j.peptides.2016.12.010

114. Pitts PM, Volkoff H. Characterization of appetite-regulating factors in platyfish, Xiphophorus maculatus (Cyprinodontiformes Poeciliidae). Comp Biochem Physiol A Mol Integr Physiol. (2017) 208:80-8. doi: 10.1016/j.cbpa.2017.03.018

115. Wei R, Zhou C, Yuan D, Wang T, Lin F, Chen H, et al. Characterization, tissue distribution and regulation of neuropeptideY in Schizothorax prenanti. J Fish Biol. (2014) 85:278-91. doi: 10.1111/jfb.12413
116. Kamijo M, Kojima K, Maruyama K, Konno N, Motohashi E, Ikegami T, et al. Neuropeptide $\mathrm{Y}$ in tiger puffer (Takifugu rubripes): distribution, cloning, characterization, and mRNA expression responses to prandial condition. Zoolog Sci. (2011) 28:882-90. doi: 10.2108/zsj.28.882

117. MacDonald E, Volkoff H. Cloning, distribution and effects of season and nutritional status on the expression of neuropeptide Y (NPY), cocaine and amphetamine regulated transcript (CART) and cholecystokinin (CCK) in winter flounder (Pseudopleuronectes americanus). Horm Behav. (2009) 56:58-65. doi: 10.1016/j.yhbeh.2009.03.002

118. MacDonald E, Volkoff H. Neuropeptide Y (NPY), cocaine- and amphetamine-regulated transcript (CART) and cholecystokinin (CCK) in winter skate (Raja ocellata): cDNA cloning, tissue distribution and mRNA expression responses to fasting. Gen Comp Endocrinol. (2009) 161:252-61. doi: 10.1016/j.ygcen.2009.01.021

119. Hosomi N, Furutani T, Takahashi N, Masumoto T, Fukada H. Yellowtail neuropeptide Y: molecular cloning, tissue distribution, and response to fasting. Fish Sci. (2014) 80:483-92. doi: 10.1007/s12562-014-0711-4

120. Yokobori E, Azuma M, Nishiguchi R, Kang KS, Kamijo M, Uchiyama M, et al. Neuropeptide Y stimulates food intake in the Zebrafish, Danio rerio. $J$ Neuroendocrinol. (2012) 24:766-73. doi: 10.1111/j.1365-2826.2012.02281.x

121. Jørgensen EH, Bernier NJ, Maule AG, Vijayan MM. Effect of long-term fasting and a subsequent meal on mRNA abundances of hypothalamic appetite regulators, central and peripheral leptin expression and plasma leptin levels in rainbow trout. Peptides. (2016) 86:162-70. doi: 10.1016/j.peptides.2015.08.010

122. Wei R, Yuan D, Wang T, Zhou C, Lin F, Chen H, et al. Characterization, tissue distribution and regulation of agouti-related protein (AgRP) in a cyprinid fish (Schizothorax prenanti). Gene. (2013) 527:193-200. doi: 10.1016/j.gene.2013.06.003

123. Agulleiro MJ, Cortés R, Leal E, Ríos D, Sánchez E, Cerdá-Reverter JM. Characterization, tissue distribution and regulation by fasting of the agouti family of peptides in the sea bass (Dicentrarchus labrax). Gen Comp Endocrinol. (2014) 205:251-9. doi: 10.1016/j.ygcen.2014.02.009

124. Wan Y, Zhang Y, Ji P, Li Y, Xu P, Sun X. Molecular characterization of CART, AgRP, and MC4R genes and their expression with fasting and refeeding in common carp (Cyprinus carpio). Mol Biol Rep. (2012) 39:2215-23. doi: 10.1007/s11033-011-0970-4

125. Song Y, Golling G, Thacker TL, Cone RD. Agouti-related protein (AGRP) is conserved and regulated by metabolic state in the zebrafish, Danio rerio. Endocrine (2003) 22:257-65. doi: 10.1385/ENDO:22:3:257

126. Kang D-Y, Kim H-C. Functional relevance of three proopiomelanocortin (POMC) genes in darkening camouflage, blind-side hypermelanosis, and appetite of Paralichthys olivaceus. Comp Biochem Physiol B Biochem Mol Biol. (2015) 179:44-56. doi: 10.1016/j.cbpb.2014.09.002

127. Cerdá-Reverter JM, Schiöth HB, Peter RE. The central melanocortin system regulates food intake in goldfish. Regul Pept. (2003) 115:101-13. doi: 10.1016/S0167-0115(03)00144-7

128. Leder EH, Silverstein JT. The pro-opiomelanocortin genes in rainbow trout (Oncorhynchus mykiss): duplications, splice variants, and differential expression. J Endocrinol. (2006) 188:355-63. doi: 10.1677/joe.1.06283

129. Liu S, Zhang C, Peng G. Effects of starvation on the expression of feeding related neuropeptides in the larval zebrafish hypothalamus. Yi Chuan Hered. (2016) 38:821-30. doi: 10.16288/j.yczz.16-087

130. Subhedar N, Barsagade VG, Singru PS, Thim L, Clausen JT. Cocaine- and amphetamine-regulated transcript peptide (CART) in the telencephalon of the catfish, Clarias gariepinus: distribution and response to fasting, 2-deoxyD-glucose, glucose, insulin, and leptin treatments. J Comp Neurol. (2011) 519:1281-300. doi: 10.1002/cne.22569

131. Nishio S-I, Gibert Y, Berekelya L, Bernard L, Brunet F, Guillot E, et al. Fasting induces CART down-regulation in the zebrafish nervous system in a cannabinoid receptor 1-dependent manner. Mol Endocrinol Baltim Md. (2012) 26:1316-26. doi: 10.1210/me.2011-1180

132. Kehoe AS, Volkoff H. Cloning and characterization of neuropeptide Y (NPY) and cocaine and amphetamine regulated transcript (CART) in Atlantic cod (Gadus morhua). Comp Biochem Physiol A Mol Integr Physiol. (2007) 146:451-61. doi: 10.1016/j.cbpa.2006.12.026

133. Kobayashi Y, Peterson BC, Waldbieser GC. Association of cocaine- and amphetamine-regulated transcript (CART) messenger RNA level, food 
intake, and growth in channel catfish. Comp Biochem Physiol A Mol Integr Physiol. (2008) 151:219-25. doi: 10.1016/j.cbpa.2008.06.029

134. Abbott M, Volkoff H. Thyrotropin Releasing Hormone (TRH) in goldfish (Carassius auratus): role in the regulation of feeding and locomotor behaviors and interactions with the orexin system and cocaine- and amphetamine regulated transcript (CART). Horm Behav. (2011) 59:236-45. doi: 10.1016/j.yhbeh.2010.12.008

135. Volkoff H. Appetite regulating peptides in red-bellied piranha, Pygocentrus nattereri: cloning, tissue distribution and effect of fasting on mRNA expression levels. Peptides. (2014) 56:116-24. doi: 10.1016/j.peptides.2014.03.022

136. Novak CM, Jiang X, Wang C, Teske JA, Kotz CM, Levine JA. Caloric restriction and physical activity in zebrafish (Danio rerio). Neurosci Lett. (2005) 383:99-104. doi: 10.1016/j.neulet.2005.03.048

137. Yokobori E, Kojima K, Azuma M, Kang KS, Maejima S, Uchiyama M, et al. Stimulatory effect of intracerebroventricular administration of orexin A on food intake in the zebrafish, Danio rerio. Peptides (2011) 32:1357-62. doi: 10.1016/j.peptides.2011.05.010

138. Kang D-Y, Kim H-C. Functional characterization of two melaninconcentrating hormone genes in the color camouflage, hypermelanosis, and appetite of starry flounder. Gen Comp Endocrinol. (2013) 189:74-83. doi: 10.1016/j.ygcen.2013.04.025

139. Tuziak SM, Volkoff H. Melanin-concentrating hormone $(\mathrm{MCH})$ and gonadotropin-releasing hormones $(\mathrm{GnRH})$ in Atlantic cod, Gadus morhua: tissue distributions, early ontogeny and effects of fasting. Peptides. (2013) 50:109-18. doi: 10.1016/j.peptides.2013.10.005

140. Takahashi A, Tsuchiya K, Yamanome T, Amano M, Yasuda A, Yamamori $\mathrm{K}$, et al. Possible involvement of melanin-concentrating hormone in food intake in a teleost fish, barfin flounder. Peptides. (2004) 25:1613-22. doi: 10.1016/j.peptides.2004.02.022

141. Mizusawa K, Amiya N, Yamaguchi Y, Takabe S, Amano M, Breves JP, et al. Identification of mRNAs coding for mammalian-type melaninconcentrating hormone and its receptors in the scalloped hammerhead shark Sphyrna lewini. Gen Comp Endocrinol. (2012) 179:78-87. doi: 10.1016/j.ygcen.2012.07.023

142. Wang T, Yuan D, Zhou C, Lin F, Wei R, Chen H, et al. Molecular characterization of melanin-concentrating hormone $(\mathrm{MCH})$ in Schizothorax prenanti: cloning, tissue distribution and role in food intake regulation. Fish Physiol Biochem. (2016) 42:883-93. doi: 10.1007/s10695-015-0182-2

143. Tuziak SM, Volkoff H. A preliminary investigation of the role of melaninconcentrating hormone $(\mathrm{MCH})$ and its receptors in appetite regulation of winter flounder (Pseudopleuronectes americanus). Mol Cell Endocrinol. (2012) 348:281-96. doi: 10.1016/j.mce.2011.09.015

144. Berman JR, Skariah G, Maro GS, Mignot E, Mourrain P. Characterization of two melanin-concentrating hormone genes in zebrafish reveals evolutionary and physiological links with the mammalian MCH system. J Comp Neurol. (2009) 517:695-710. doi: 10.1002/cne.22171

145. Hevrøy EM, Azpeleta C, Shimizu M, Lanzén A, Kaiya H, Espe M, et al. Effects of short-term starvation on ghrelin, GH-IGF system, and IGFbinding proteins in Atlantic salmon. Fish Physiol Biochem. (2011) 37:217-32. doi: 10.1007/s10695-010-9434-3

146. Feng K, Zhang G-R, Wei K-J, Xiong B-X. Molecular cloning, tissue distribution, and ontogenetic expression of ghrelin and regulation of expression by fasting and refeeding in the grass carp (Ctenopharyngodon idellus). J Exp Zool Part Ecol Genet Physiol. (2013) 319:202-12. doi: $10.1002 /$ jez.1784

147. Eom J, Hong M, Cone RD, Song Y. Zebrafish ghrelin is expressed in pancreatic endocrine cells and regulated by metabolic state. Biochem Biophys Res Commun. (2013) 439:115-20. doi: 10.1016/j.bbrc.2013.08.017

148. Amole N, Unniappan S. Fasting induces preproghrelin mRNA expression in the brain and gut of zebrafish, Danio rerio. Gen Comp Endocrinol. (2009) 161:133-7. doi: 10.1016/j.ygcen.2008.11.002

149. Murashita K, Kurokawa T, Nilsen TO, Rønnestad I. Ghrelin, cholecystokinin, and peptide YY in Atlantic salmon (Salmo salar): molecular cloning and tissue expression. Gen Comp Endocrinol. (2009) 160:223-35. doi: 10.1016/j.ygcen.2008.11.024

150. Song Y, Zhao C, Liang X-F, He S, Tian C, Cheng X, et al. Effects of fasting, temperature, and photoperiod on preproghrelin mRNA expression in Chinese perch. Fish Physiol Biochem. (2017) 43:803-12. doi: 10.1007/s10695-016-0335-y

151. Zhou C, Zhang X, Liu T, Wei R, Yuan D, Wang T, et al. Schizothorax davidi ghrelin: cDNA cloning, tissue distribution and indication for its stimulatory character in food intake. Gene. (2014) 534:72-7. doi: $10.1016 /$ j.gene.2013.10.012

152. Terova G, Rimoldi S, Bernardini G, Gornati R, Saroglia M. Sea bass ghrelin: molecular cloning and mRNA quantification during fasting and refeeding. Gen Comp Endocrinol. (2008) 155:341-51. doi: 10.1016/j.ygcen.2007.05.028

153. Koven W, Schulte $P$. The effect of fasting and refeeding on mRNA expression of PepT1 and gastrointestinal hormones regulating digestion and food intake in zebrafish (Danio rerio). Fish Physiol Biochem. (2012) 38:1565-75. doi: 10.1007/s10695-012-9649-6

154. Picha ME, Strom CN, Riley LG, Walker AA, Won ET, Johnstone WM, et al. Plasma ghrelin and growth hormone regulation in response to metabolic state in hybrid striped bass: effects of feeding, ghrelin and insulin-like growth factor-I on in vivo and in vitro GH secretion. Gen Comp Endocrinol. (2009) 161:365-72. doi: 10.1016/j.ygcen.2009.01.026

155. Riley LG, Fox BK, Breves JP, Kaiya H, Dorough CP, Hirano T, et al. Absence of effects of short-term fasting on plasma ghrelin and brain expression of ghrelin receptors in the tilapia, Oreochromis mossambicus. Zoolog Sci. (2008) 25:821-7. doi: 10.2108/zsj.25.821

156. Matsuda K, Miura T, Kaiya H, Maruyama K, Uchiyama M, Kangawa $\mathrm{K}$, et al. Stimulatory effect of n-octanoylated ghrelin on locomotor activity in the goldfish, Carassius auratus. Peptides. (2006) 27:1335-40. doi: 10.1016/j.peptides.2005.10.011

157. Volkoff H. Cloning, tissue distribution and effects of fasting on mRNA expression levels of leptin and ghrelin in red-bellied piranha (Pygocentrus nattereri). Gen Comp Endocrinol. (2015) 217-218:20-27. doi: 10.1016/j.ygcen.2015.05.004

158. Feng K, Zhang G, Wei K, Xiong B, Liang T, Ping H. Molecular characterization of cholecystokinin in grass carp (Ctenopharyngodon idellus): cloning, localization, developmental profile, and effect of fasting and refeeding on expression in the brain and intestine. Fish Physiol Biochem. (2012) 38:1825-34. doi: 10.1007/s10695-0129679-0

159. Micale V, Campo S, D'Ascola A, Guerrera MC, Levanti MB, German,à A, et al. Cholecystokinin in white sea bream: molecular cloning, regional expression, and immunohistochemical localization in the gut after feeding and fasting. PLoS ONE. (2012) 7:e52428. doi: 10.1371/journal.pone.00 52428

160. Murashita K, Fukada H, Hosokawa H, Masumoto T. Cholecystokinin and peptide Y in yellowtail (Seriola quinqueradiata): molecular cloning, realtime quantitative RT-PCR, and response to feeding and fasting. Gen Comp Endocrinol. (2006) 145:287-97. doi: 10.1016/j.ygcen.2005.09.008

161. Huising MO, Geven EJW, Kruiswijk CP, Nabuurs SB, Stolte EH, Spanings FAT, et al. Increased leptin expression in common Carp (Cyprinus carpio) after food intake but not after fasting or feeding to satiation. Endocrinology. (2006) 147:5786-97. doi: 10.1210/en.2006-0824

162. Fuentes EN, Kling P, Einarsdottir IE, Alvarez M, Valdés JA, Molina A, et al. Plasma leptin and growth hormone levels in the fine flounder (Paralichthys adspersus) increase gradually during fasting and decline rapidly after refeeding. Gen Comp Endocrinol. (2012) 177:120-7. doi: 10.1016/j.ygcen.2012.02.019

163. Fuentes EN, Safian D, Einarsdottir IE, Valdés JA, Elorza AA, Molina A, et al. Nutritional status modulates plasma leptin, AMPK and TOR activation, and mitochondrial biogenesis: implications for cell metabolism and growth in skeletal muscle of the fine flounder. Gen Comp Endocrinol. (2013) 186:17280. doi: 10.1016/j.ygcen.2013.02.009

164. Kling P, Rønnestad I, Stefansson SO, Murashita K, Kurokawa T, Björnsson BT. A homologous salmonid leptin radioimmunoassay indicates elevated plasma leptin levels during fasting of rainbow trout. Gen Comp Endocrinol. (2009) 162:307-12. doi: 10.1016/j.ygcen.2009.04.003

165. Zhang H, Chen H, Zhang Y, Li S, Lu D, Zhang H, et al. Molecular cloning, characterization and expression profiles of multiple leptin genes and a leptin receptor gene in orange-spotted grouper (Epinephelus coioides). Gen Comp Endocrinol. (2013) 181:295-305. doi: 10.1016/j.ygcen.2012. 09.008 
166. Shpilman M, Hollander-Cohen L, Ventura T, Gertler A, Levavi-Sivan B. Production, gene structure and characterization of two orthologs of leptin and a leptin receptor in tilapia. Gen Comp Endocrinol. (2014) 207:74-85. doi: 10.1016/j.ygcen.2014.05.006

167. Gambardella C, Gallus L, Amaroli A, Terova G, Masini MA, Ferrando S. Fasting and re-feeding impact on leptin and aquaglyceroporin 9 in the liver of European sea bass (Dicentrarchus labrax). Aquaculture. (2012) 354-5:1-6. doi: 10.1016/j.aquaculture.2012.04.043

168. Johnson RM, Johnson TM, Londraville RL. Evidence for leptin expression in fishes. J Exp Zool. (2000) 286:718-24. doi: 10.1002/(SICI)1097010X(20000601)286:7<718::AID-JEZ6>3.0.CO;2-I

169. Johansson M, Morgenroth D, Einarsdottir IE, Gong N, Björnsson BT. Energy stores, lipid mobilization and leptin endocrinology of rainbow trout. J Comp Physiol B. (2016) 186:759-73. doi: 10.1007/s00360-016-0988-y

170. Won ET, Baltzegar DA, Picha ME, Borski RJ. Cloning and characterization of leptin in a Perciform fish, the striped bass (Morone saxatilis): control of feeding and regulation by nutritional state. Gen Comp Endocrinol. (2012) 178:98-107. doi: 10.1016/j.ygcen.2012.04.019

171. Chen T, Chen S, Ren C, Hu C, Tang D, Yan A. Two isoforms of leptin in the White-clouds Mountain minnow (Tanichthys albonubes): differential regulation by estrogen despite similar response to fasting. Gen Comp Endocrinol. (2016) 225:174-84. doi: 10.1016/j.ygcen.2015.08.002

172. Gorissen M, Bernier NJ, Nabuurs SB, Flik G, Huising MO. Two divergent leptin paralogues in zebrafish (Danio rerio) that originate early in teleostean evolution. J Endocrinol. (2009) 201:329-39. doi: 10.1677/JOE-09-0034

173. Jørgensen EH, Martinsen M, Strøm V, Hansen KER, Ravuri CS, Gong N, et al. Long-term fasting in the anadromous Arctic charr is associated with downregulation of metabolic enzyme activity and upregulation of leptin A1 and SOCS expression in the liver. J Exp Biol. (2013) 216:3222-30. doi: $10.1242 /$ jeb.088344

174. Morini M, Pasquier J, Dirks R, van den Thillart G, Tomkiewicz J, Rousseau $\mathrm{K}$, et al. Duplicated leptin receptors in two species of eel bring new insights into the evolution of the leptin system in vertebrates. PLOS ONE. (2015) 10:e0126008. doi: 10.1371/journal.pone.0126008

175. Narnaware YK, Peter RE. Influence of diet composition on food intake and neuropeptide Y (NPY) gene expression in goldfish brain. Regul Pept. (2002) 103:75-83. doi: 10.1016/S0167-0115(01)00342-1

176. Blanco AM, Bertucci JI, Delgado MJ, Valenciano AI, Unniappan S. Tissue-specific expression of ghrelinergic and NUCB2/nesfatin-1 systems in goldfish (Carassius auratus) is modulated by macronutrient composition of diets. Comp Biochem Physiol A Mol Integr Physiol. (2016) 195:1-9. doi: 10.1016/j.cbpa.2016.01.016

177. Bertucci JI, Blanco AM, Canosa LF, Unniappan S. Glucose, amino acids and fatty acids directly regulate ghrelin and NUCB2/nesfatin-1 in the intestine and hepatopancreas of goldfish (Carassius auratus) in vitro. Comp Biochem Physiol A Mol Integr Physiol. (2017) 206:24-35. doi: 10.1016/j.cbpa.2017.01.006

178. Babaei S, Sáez A, Caballero-Solares A, Fernández F, Baanante IV, Metón I. Effect of dietary macronutrients on the expression of cholecystokinin, leptin, ghrelin and neuropeptide Y in gilthead sea bream (Sparus aurata). Gen Comp Endocrinol. (2017) 240:121-8. doi: 10.1016/j.ygcen.2016.10.003

179. Bertucci JI, Tovar MO, Unniappan S, Navarro JC, Canosa LF. Effects of dietary sunflower oil on growth parameters, fatty acid profiles and expression of genes regulating growth and metabolism in the pejerrey (Odontesthes bonariensis) fry. Aquac. Nutr. (2017) 24:748-57. doi: 10.1111/anu. 12603

180. Bonacic K, Campoverde C, Gómez-Arbonés J, Gisbert E, Estevez A, Morais S. Dietary fatty acid composition affects food intake and gutbrain satiety signaling in Senegalese sole (Solea senegalensis, Kaup 1858) larvae and post-larvae. Gen Comp Endocrinol. (2016) 228:79-94. doi: 10.1016/j.ygcen.2016.02.002

181. Moriyama S, Ayson FG, Kawauchi H. Growth regulation by insulinlike growth factor-I in fish. Biosci Biotechnol Biochem. (2000) 64:1553-62. doi: 10.1271/bbb.64.1553

182. Pierce AL, Shimizu M, Beckman BR, Baker DM, Dickhoff WW. Time course of the GH/IGF axis response to fasting and increased ration in chinook salmon (Oncorhynchus tshawytscha). Gen Comp Endocrinol. (2005) 140:192-202. doi: 10.1016/j.ygcen.2004.10.017
183. Small BC, Peterson BC. Establishment of a time-resolved fluoroimmunoassay for measuring plasma insulin-like growth factor I (IGF-I) in fish: effect of fasting on plasma concentrations and tissue mRNA expression of IGF-I and growth hormone (GH) in channel catfish (Ictalurus punctatus). Domest Anim Endocrinol. (2005) 28:202-15. doi: 10.1016/j.domaniend.2004.09.002

184. Shimizu M, Cooper KA, Dickhoff WW, Beckman BR. Postprandial changes in plasma growth hormone, insulin, insulin-like growth factor (IGF)-I, and IGF-binding proteins in coho salmon fasted for varying periods. Am J Physiol Regul Integr Comp Physiol. (2009) 297:R352-361. doi: 10.1152/ajpregu.90939.2008

185. Cleveland BM, Weber GM, Blemings KP, Silverstein JT. Insulin-like growth factor-I and genetic effects on indexes of protein degradation in response to feed deprivation in rainbow trout (Oncorhynchus mykiss). Am J Physiol Regul Integr Comp Physiol. (2009) 297:R1332-42. doi: 10.1152/ajpregu.00272.2009

186. Farbridge KJ, Leatherland JF. Temporal changes in plasma thyroid hormone, growth hormone and free fatty acid concentrations, and hepatic 5'monodeiodinase activity, lipid and protein content during chronic fasting and re-feeding in rainbow trout (Oncorhynchus mykiss). Fish Physiol Biochem. (1992) 10:245-57. doi: 10.1007/BF00004518

187. Johnsson JI, Jönsson E, Björnsson BT. Dominance, nutritional state, and growth hormone levels in rainbow trout (Oncorhynchus mykiss). Horm Behav. (1996) 30:13-21. doi: 10.1006/hbeh.1996.0003

188. Fox BK, Riley LG, Hirano T, Grau EG. Effects of fasting on growth hormone, growth hormone receptor, and insulin-like growth factor-I axis in seawateracclimated tilapia, Oreochromis mossambicus. Gen Comp Endocrinol. (2006) 148:340-7. doi: 10.1016/j.ygcen.2006.04.007

189. Uchida K, Kajimura S, Riley LG, Hirano T, Aida K, Grau EG. Effects of fasting on growth hormone/insulin-like growth factor I axis in the tilapia, Oreochromis mossambicus. Comp Biochem Physiol A Mol Integr Physiol. (2003) 134:429-39. doi: 10.1016/S1095-6433(02)00 318-5

190. Delgadin TH, Pérez Sirkin DI, Di Yorio MP, Arranz SE, Vissio PG. GH, IGF-I and GH receptors mRNA expression in response to growth impairment following a food deprivation period in individually housed cichlid fish Cichlasoma dimerus. Fish Physiol Biochem. (2015) 41:51-60. doi: 10.1007/s10695-0140005-x

191. Zhong H, Zhou Y, Liu S, Tao M, Long Y, Liu Z, et al. Elevated expressions of GH/IGF axis genes in triploid crucian carp. Gen Comp Endocrinol. (2012) 178:291-300. doi: 10.1016/j.ygcen.2012.06.006

192. Pedroso FL, de Jesus-Ayson EGT, Cortado HH, Hyodo S, Ayson FG. Changes in mRNA expression of grouper (Epinephelus coioides) growth hormone and insulin-like growth factor I in response to nutritional status. Gen Comp Endocrinol. (2006) 145:237-46. doi: 10.1016/j.ygcen.2005. 09.001

193. Fox BK, Breves JP, Davis LK, Pierce AL, Hirano T, Grau EG. Tissuespecific regulation of the growth hormone/insulin-like growth factor axis during fasting and re-feeding: Importance of muscle expression of IGF-I and IGF-II mRNA in the tilapia. Gen Comp Endocrinol. (2010) 166:573-80. doi: 10.1016/j.ygcen.2009.11.012

194. Fukada H, Murashita K, Furutani T, Masumoto T. Yellowtail insulinlike growth factor 1: molecular cloning and response to various nutritional conditions. Domest Anim Endocrinol. (2012) 42:220-9. doi: 10.1016/j.domaniend.2011.12.005

195. Baños N, Planas JV, Gutiérrez J, Navarro I. Regulation of plasma insulin-like growth factor-I levels in brown trout (Salmo trutta). Comp Biochem Physiol C Pharmacol Toxicol Endocrinol. (1999) 124:33-40. doi: 10.1016/S0742-8413(99)00044-4

196. Kawaguchi K, Kaneko N, Fukuda M, Nakano Y, Kimura S, Hara A, et al. Responses of insulin-like growth factor (IGF)-I and two IGFbinding protein-1 subtypes to fasting and re-feeding, and their relationships with individual growth rates in yearling masu salmon (Oncorhynchus masou). Comp Biochem Physiol A Mol Integr Physiol. (2013) 165:191-8. doi: 10.1016/j.cbpa.2013.02.029

197. Pérez-Sánchez J, Le Bail P-Y. Growth hormone axis as marker of nutritional status and growth performance in fish. Aquaculture. (1999) 177:117-28. doi: 10.1016/S0044-8486(99)00073-3 
198. Dyer AR, Barlow CG, Bransden MP, Carter CG, Glencross BD, Richardson $\mathrm{N}$, et al. Correlation of plasma IGF-I concentrations and growth rate in aquacultured finfish: a tool for assessing the potential of new diets. Aquaculture. (2004) 236:583-92. doi: 10.1016/j.aquaculture.2003.12.025

199. Pérez-Sánchez J, Martí-Palanca H, Kaushik SJ. Nutrient requirements and interactions ration size and protein intake affect circulating growth hormone concentration, hepatic growth hormone binding and plasma insulin-like growth factor-I immunoreactivity in a marine teleost, the gilthead sea bream (Sparus aurata). J. Nutr. (1995) 546-52.

200. Picha ME, Turano MJ, Beckman BR, Borski RJ. Endocrine biomarkers of growth and applications to aquaculture: a minireview of growth hormone, insulin-like growth factor (IGF)-I, and IGF-binding proteins as potential growth indicators in fish. N Am J Aquac. (2008) 70:196-211. doi: 10.1577/A07-038.1

201. Rodgers BD, Helms LM, Grau EG. Effects of fasting, medium glucose, and amino acid concentrations on prolactin and growth hormone release, in vitro, from the pituitary of the tilapia Oreochromis mossambicus. Gen Comp Endocrinol. (1992) 86:344-351.

202. Riley LG, Walker AP, Dorough CP, Schwandt SE, Grau EG. Glucose regulates ghrelin, neuropeptide Y, and the GH/IGF-I axis in the tilapia, Oreochromis mossambicus. Comp Biochem Physiol A Mol Integr Physiol. (2009) 154:541-6. doi: 10.1016/j.cbpa.2009.08.018

203. Bertucci JI, Blanco AM, Canosa LF. Direct actions of macronutrient components on goldfish hepatopancreas in vitro to modulate the expression of ghr-I, ghr-II, igf-I and igf-II mRNAs. Gen Comp Endocrinol. (2017) 250:1-8. doi: 10.1016/j.ygcen.2017.05.014

204. Yakar S, Setser J, Zhao H, Stannard B, Haluzik M, Glatt V, et al. Inhibition of growth hormone action improves insulin sensitivity in liver IGF-1 -deficient mice. J Clin Invest. (2004) 113:96. doi: 10.1172/JCI200417763.Introduction

205. Wood AW, Duan C, Bern HA. Insulin-like growth factor signaling in fish. Int Rev Cytol. (2005) 243:215-85. doi: 10.1016/S0074-7696(05)43004-1

206. Ekström K, Pulkkinen MA, Carlsson-Skwirut C, Brorsson AL, Ma Z, Frystyk J, et al. Tissue IGF-I measured by microdialysis reflects body glucose utilization after rhIGF-I injection in type 1 diabetes. J Clin Endocrinol Metab. (2015) 100:4299-306. doi: 10.1210/jc.2015-2070

207. Baumann MU, Schneider H, Malek A, Palta V, Surbek DV, Sager R, et al. Regulation of human trophoblast GLUT1 glucose transporter by Insulin-Like Growth Factor I (IGF-I). PLoS ONE. (2014) 9:e106037. doi: 10.1371/journal.pone.0106037

208. Sangiao-Alvarellos S, Míguez JM, Soengas JL. Actions of growth hormone on carbohydrate metabolism and osmoregulation of rainbow trout (Oncorhynchus mykiss). Gen Comp Endocrinol. (2005) 141:214-25. doi: 10.1016/j.ygcen.2005.01.007

209. Huang JF, Xu QY, Chang YM. Effects of temperature and dietary protein on the growth performance and IGF-I mRNA expression of juvenile mirror carp (Cyprinus carpio). Aquac Nutr. (2016) 22:283-92. doi: 10.1111/anu. 12254

210. Qiang J, Yang H, Wang H, Kpundeh MD, Xu P. Growth and IGF-I response of juvenile Nile tilapia (Oreochromis niloticus) to changes in water temperature and dietary protein level. J Therm Biol. (2012) 37:686-95. doi: 10.1016/j.jtherbio.2012.07.009

211. Tu Y, Xie S, Han D, Yang Y, Jin J, Liu H, et al. Growth performance, digestive enzyme, transaminase and GH-IGF-I axis gene responsiveness to different dietary protein levels in broodstock allogenogynetic gibel carp (Carassius auratus gibelio) CAS III. Aquaculture. (2015) 446:290-7. doi: 10.1016/j.aquaculture.2015.05.003

212. Borges P, Oliveira B, Casal S, Dias J, Conceição L, Valente LMP. Dietary lipid level affects growth performance and nutrient utilisation of Senegalese sole (Solea senegalensis) juveniles. Br J Nutr. (2009) 102:1007-14. doi: $10.1017 /$ S0007114509345262
213. Regost C, Arzel J, Cardinal M, Robin J, Laroche M, Kaushik SJ. Dietary lipid level, hepatic lipogenesis and flesh quality. Nutrition. (2001) 193:291-309. doi: 10.1016/S0044-8486(00)00493-2

214. Lee S-M, Cho SH, Kim K-D. Effects of dietary protein and energy levels on growth and body composition of juvenile flounder Paralichthys olivaceus. J World Aquac Soc. (2000) 31:306-315. doi: 10.1111/j.1749-7345.2000.tb00882.x

215. Campos C, Valente LMP, Borges P, Bizuayehu T, Fernandes JMO. Dietary lipid levels have a remarkable impact on the expression of growth-related genes in Senegalese sole (Solea senegalensis Kaup). J Exp Biol. (2010) 213:2009. doi: 10.1242/jeb.033126

216. Gómez-Requeni P, Kraemer MN, Canosa LF. Regulation of somatic growth and gene expression of the GH-IGF system and PRP-PACAP by dietary lipid level in early juveniles of a teleost fish, the pejerrey (Odontesthes bonariensis). J Comp Physiol B. (2012) 182:517-30. doi: 10.1007/s00360-0110640-9

217. Chen N, Jin L, Zhou H, Qiu X. Effects of dietary arginine levels and carbohydrate-to-lipid ratios on mRNA expression of growth-related hormones in largemouth bass, Micropterus salmoides. Gen Comp Endocrinol. (2012) 179:121-7. doi: 10.1016/j.ygcen.2012.08.004

218. Benedito-Palos L, Saera-Vila A, Calduch-Giner JA, Kaushik S, Pérez-Sánchez J. Combined replacement of fish meal and oil in practical diets for fast growing juveniles of gilthead sea bream (Sparus aurata L.): networking of systemic and local components of GH/IGF axis. Aquaculture. (2007) 267:199-212. doi: 10.1016/j.aquaculture.2007.01.011

219. Mazurais D, Darias MJ, Gouillou-Coustans MF, Le Gall MM, Huelvan C, Desbruyères E, et al. Dietary vitamin mix levels influence the ossification process in European sea bass (Dicentrarchus labrax) larvae. Am J Physiol Regul Integr Comp Physiol. (2008) 294:R520-7. doi: 10.1152/ajpregu.00659.2007

220. Bergan-Roller HE, Sheridan MA. The growth hormone signaling system: insights into coordinating the anabolic and catabolic actions of growth hormone. Gen Comp Endocrinol. (2017) 258:119-33. doi: 10.1016/j.ygcen.2017.07.028

221. Unniappan S, Lin X, Cervini L, Rivier J, Kaiya H, Kangawa K, et al. Goldfish ghrelin: Molecular characterization of the complementary deoxyribonucleic acid, partial gene structure and evidence for its stimulatory role in food intake. Endocrinology. (2002) 143:4143-6. doi: 10.1210/en.2002-220644

222. Unniappan S, Peter RE. In vitro and in vivo effects of ghrelin on luteinizing hormone and growth hormone release in goldfish. Am J Physiol Regul Integr Comp Physiol. (2004) 286:R1093-101. doi: 10.1152/ajpregu.00669.2003

223. Grey CL, Chang JP. Ghrelin-induced growth hormone release from goldfish pituitary cells is nitric oxide dependent. Gen Comp Endocrinol. (2012) 179:152-8. doi: 10.1016/j.ygcen.2012.08.012

224. Won ET, Douros JD, Hurt DA, Borski RJ. Leptin stimulates hepatic growth hormone receptor and insulin-like growth factor gene expression in a teleost fish, the hybrid striped bass. Gen Comp Endocrinol. (2016) 229:84-91. doi: 10.1016/j.ygcen.2016.02.003

Conflict of Interest Statement: The authors declare that the research was conducted in the absence of any commercial or financial relationships that could be construed as a potential conflict of interest.

Copyright (c) 2019 Bertucci, Blanco, Sundarrajan, Rajeswari, Velasco and Unniappan. This is an open-access article distributed under the terms of the Creative Commons Attribution License (CC BY). The use, distribution or reproduction in other forums is permitted, provided the original author(s) and the copyright owner(s) are credited and that the original publication in this journal is cited, in accordance with accepted academic practice. No use, distribution or reproduction is permitted which does not comply with these terms. 